\title{
37. HISTORY OF PLIO-PLEISTOCENE CLIMATE IN THE NORTHEASTERN ATLANTIC, DEEP SEA DRILLING PROJECT HOLE 552A ${ }^{1}$
}

\author{
H. B. Zimmerman, Union College \\ N. J. Shackleton, Cambridge University \\ J. Backman, University of Stockholm \\ D. V. Kent, Lamont-Doherty Geological Observatory \\ J. G. Baldauf, U. S. Geological Survey \\ A. J. Kaltenback, Marathon Oil Co. \\ and \\ A. C. Morton, British Geological Survey, Keyworth ${ }^{2}$
}

\begin{abstract}
DSDP Hole 552A, cored with the HPC on Hatton Drift, represents an almost complete and undisturbed sediment section spanning the late Neogene and Quaternary. Lithologic, faunal, isotopic, and paleomagnetic analyses indicate that the section represents the most complete deep sea record of climatic evolution hitherto recovered at high latitudes in the northern hemisphere. A glacial record of remarkable resolution for the late Pliocene and Pleistocene is provided by oxygen and carbon isotope ratios in benthic foraminifers. In the upper part of the section, the whole of the standard oxygen isotope record of the past million years is well preserved. The onset of ice-rafting and glacial-interglacial alternations occurs at about 2.4 m.y. ago.
\end{abstract}

\section{INTRODUCTION}

The introduction of the hydraulic piston corer (HPC) by the Deep Sea Drilling Project (DSDP) has provided, for the first time, the ability to retrieve virtually undisturbed sections of Neogene sediment from the deep ocean. In HPC Hole 552A, we obtained an almost complete record of the inception of glaciation and subsequent variation of Plio-Pleistocene climate in a high latitude region of the North Atlantic (Figs. 1, 2). In this chapter we summarize the sedimentologic, faunal, geochemical, paleomagnetic, and isotopic data generated by examination of this core.

A relatively high rate of sediment accumulation at HPC Hole 552A provided an unusual and detailed PlioPleistocene record with good resolution and minimal bioturbation. In contrast, relatively few conventional piston cores, such as core V28-239, contain a complete record of glacial stratigraphy because bioturbation and low rates of accumulation obscure the fine-scale resolution of detailed features of the climatic record.

In Hole 552A deposition is fashioned by the interplay of two climatically controlled processes: the influx of noncarbonate material transported primarily by ice-rafting and bottom currents and the changing production of calcareous microfossils in the surface waters (carbonate dissolution is of little importance at the water depths of

\footnotetext{
${ }^{1}$ Roberts, D. G., Schnitker, D., et al., Init. Repts. DSDP, 81: Washington (U.S. Govt. Printing Office).

2 Addresses: (Zimmerman) Union College, Schenectady, New York; (Shackleton) Godwin Laboratory for Quaternary Research, Cambridge University, Cambridge CB2.3 RS, United Kingdom; (Backman) Department of Geology, University of Stockholm, Stockholm, S-106 91, Sweden; (Kent) Lamont-Doherty Geological Observatory, Palisades, NY 10964; (Baldauf) U.S. Geological Survey, Menlo Park, CA 94025; (Kaltenback) Marathon Oil Company, Denver Research Center, Littleton, CO 80160; (Morton), British Geological Survey, Keyworth NG12 5GG, United Kingdom.
}

this site). The section is thus characterized by a striking alternation of biogenic ooze and terrigenous muds. A uniform rate of sedimentation is certainly unlikely with so dramatic a variation in the nature of the sediments. Although depositional processes are variable, the core nevertheless exhibits a remarkably complete stratigraphic record.

\section{METHODS}

All cores from Hole 552A were extremely well preserved (see Site 552 chapter) with the exception of Core 6 (24-29 m sub-bottom). Because of severe disturbance, Core 6 was excluded from all analyses.

Total carbonate content was determined by the "Karbonate Bombe" technique (Müller and Gastner, 1971). In this procedure, a sample is powdered, weighed, and treated with $6 \mathrm{~N} \mathrm{HCl}$ in a closed cylinder. The resulting $\mathrm{CO}_{2}$ pressure is proportional to the $\mathrm{CaCO}_{3}$ content of the sample. Application of an appropriate calibration factor to the manometer reading yields percent $\mathrm{CaCO}_{3}$. For sediments rich in $\mathrm{CaCO}_{3}$, error is generally as low as $1 \%$. Repeated analyses show that an accuracy of $\pm 2 \%$ is obtained for samples with $\mathrm{CaCO}_{3}$ content below $10 \%$. Analyses were made on the whole sediment and on the coarse fraction (CF) after screening at $74 \mu \mathrm{m}$ (Table 1, Fig. 3).

Following carbonate determination, the remaining acid-insoluble fraction of selected samples was examined petrographically (Morton, this volume). Cores 1 through 10 were examined for major compositional variation. A closely spaced sample set for Cores 1 and 2 was examined for the abundance of volcanic glass. A small number of samples were also analyzed for heavy mineral content, following separation from the light minerals by gravity-settling in bromoform (sp. grav. 2.80).

Organic geochemical and visual kerogen analysis was performed on selected samples covering two glacial-interglacial cycles from near the top and bottom of the section. Sedimentary organic matter was evaluated by Rock-Eval pyrolysis, determination of organic phosphorous, and pyrolysis/mass spectrometry (Figs. 4, 5). A complete description of these methodologies and results is given in Kaltenback et al. (this volume).

Diatom biostratigraphy in the Plio-Pleistocene section was established by Baldauf (this volume). Values of species abundance were based on the average number of diatom frustules observed per field of view (at least 450 fields of view per sample; $0.5 \mathrm{~mm}^{2}$ at $500 \times$ ). 


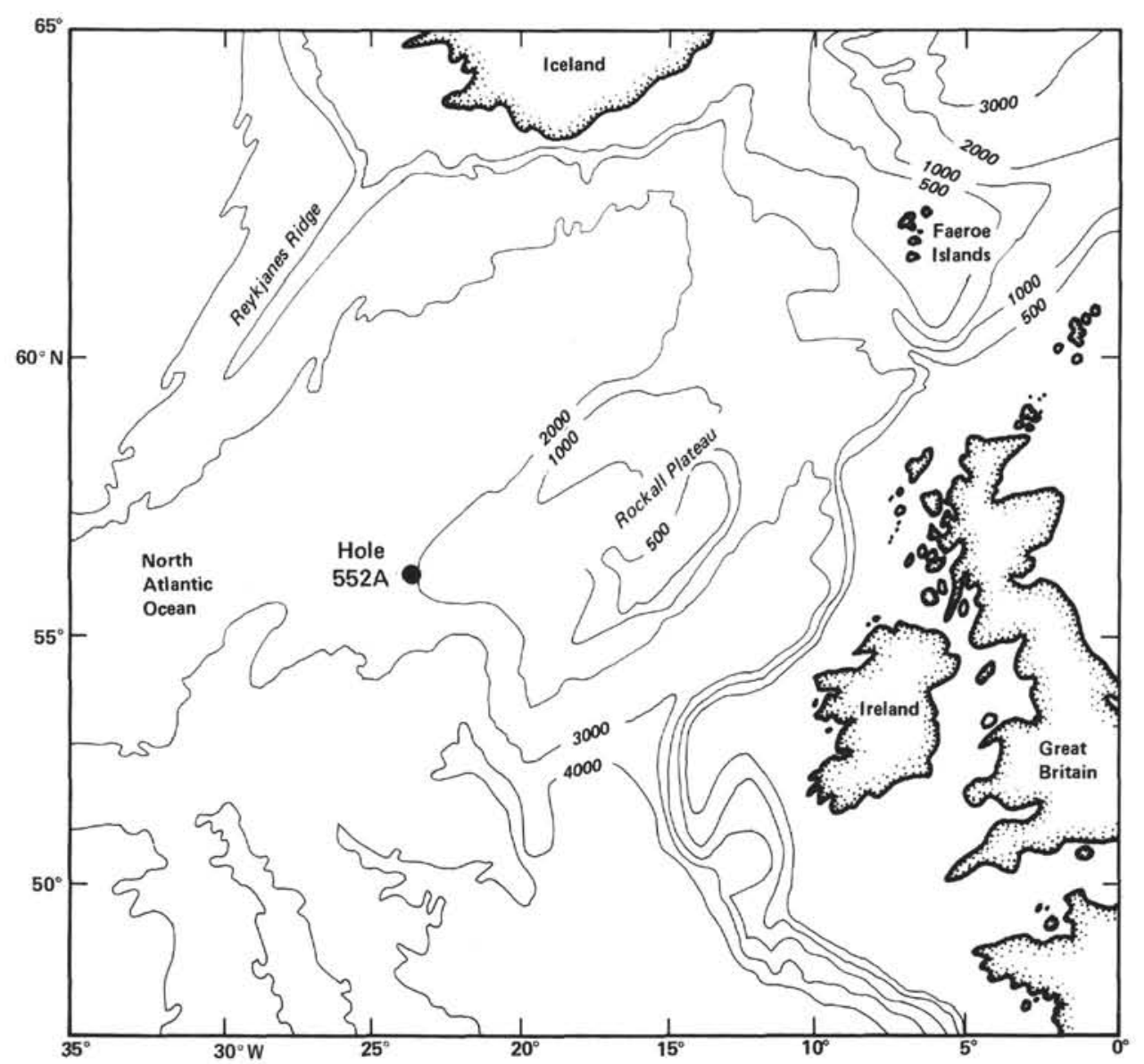

Figure 1. Location map for Hole $552 \mathrm{~A}\left(56^{\circ} 02.56^{\prime} \mathrm{N}, 23^{\circ} 13.88^{\prime} \mathrm{W}\right.$; water depth: $\left.2311 \mathrm{~m}\right)$.

Samples for paleomagnetic analysis were taken approximately every $25 \mathrm{~cm}$; we avoided sediment section that showed any sign of disturbance. Only orientation with respect to the vertical axis was preserved so that the inclination component was used to infer polarity at this high-latitude site. Natural remanent magnetization (NRM) was measured on a two-axis cryogenic magnetometer (Goree and Fuller, 1976) both before and after alternating-field demagnetization at 10 to $30 \mathrm{mT}$. NRM intensities are typically of the order $10^{-2} \mathrm{~A} / \mathrm{m}$ in the upper $45 \mathrm{~m}$ of the section, decreasing to about $10^{-3} \mathrm{~A} / \mathrm{m}$ in the lower part. Inclinations are generally well grouped near to the expected dipole valve (71 degrees, positive for normal and negative for reversed) for the latitude of the site. A reliable record of the geomagnetic field was obtained to the base of the Gauss normal magnetochron (Fig. 6). The lower boundary of the Olduvai subchron is obscured by disturbance at the break between successive cores, while the Mammoth subchron is unclear, probably lying in a slightly disturbed section at the top of Core 11 .

Stable oxygen and carbon isotope analyses were made at intervals of approximately $10 \mathrm{~cm}$ from well below the first ice-rafting layer through to the recent sediment (Figs. 7-9). The sample was dispersed by shaking for a few hours in distilled water, and sieved on a $150 \mu \mathrm{m}$ screen. The portion retained was dried at $60^{\circ} \mathrm{C}$ and weighed; the fine fraction was settled for $24 \mathrm{hr}$., dried, and weighed; the residue was retained for nannofossil studies (Backman, this volume). Three species were utilized for stable isotope analysis: Globocassidulina subglobosa, Uvigerina peregrina, and Planulina wuellerstorfi. Standard techniques for analysis were used: reaction with $100 \%$ orthophosphoric acid at $50^{\circ} \mathrm{C}$, removal of water, and isotopic analysis in a VG Isogas 903 triple collector mass spectrometer. Overall analytical uncertainty during the time these measurements were made was $\pm 0.07 \%(0-18)$ and $\pm 0.05 \%$ (C13) (both 1-sigma valves). A more complete description of these techniques and tables of analytical results and calibrations are given in Shackleton et al. (1984) and Shackleton and Hall (this volume).

\section{LITHOLOGIC DESCRIPTION}

Hole $552 \mathrm{~A}$ is located at a water depth of $2311 \mathrm{~m}$ on the Hatton Sediment Drift at the base of the western flank of Rockall Plateau (Site 552 chapter, Figs. 1, 2). The drift sediments are thought to have been deposited under bottom currents flowing northeastward unparallel to the slope. According to McCave et al. (1980), this flow consists of Iceland-Scotland Overflow Water which has rounded the southern end of the plateau after having passed over Feni Drift in the Rockall Trough.

The uppermost sediments are Plio-Pleistocene in age and are characterized by alternating beds of foraminiferal-nannofossil ooze and calcareous marls and mud. These extend from the seafloor to a basal contact defined by the lowermost marl in this unit (Section 552A-9-4; $43.6 \mathrm{~m}$ sub-bottom), which is also defined by the base of high magnetic intensity. This part of the section was continuously cored with the HPC; with the exception of Core 6 (24-29 m sub-bottom), most of the cores were undisturbed, as indicated by the well-defined stratigraphic contacts and sedimentological details.

One of the most striking characteristics of this unit is the cyclicity of color and carbonate content (Fig. 7A, B). Color cycles are clearly coupled with carbonate content, the lighter shades correlating with high carbonate values. In this part of the North Atlantic, variation in 
Table 1. Calcium carbonate data, Hole 552A.

\begin{tabular}{|c|c|c|c|c|}
\hline Depth (m) & $\begin{array}{l}\text { Core-Section } \\
\text { (level in } \mathrm{cm} \text { ) }\end{array}$ & $\mathrm{CaCO}_{3}(\%)$ & CF $(\%)$ & $\begin{array}{c}\mathrm{CF} \\
\mathrm{CaCO}_{3}(\%)\end{array}$ \\
\hline 0.08 & $1-1,7$ & 80 & 44 & 85 \\
\hline 0.16 & $1-1,15$ & 82 & 62 & 90 \\
\hline 0.21 & $1-1,20$ & 76 & 51 & 84 \\
\hline 0.31 & $1-1,30$ & 52 & 41 & 66 \\
\hline 0.39 & $1-1,38$ & 34 & 23 & 69 \\
\hline 0.49 & $1-1,48$ & 34 & & \\
\hline 0.61 & $1-1,60$ & 28 & 27 & 56 \\
\hline 0.69 & $1-1,68$ & 20 & 16 & 62 \\
\hline 0.80 & $1-1,79$ & 33 & 18 & 77 \\
\hline 0.91 & $1-1,90$ & 37 & 29 & 64 \\
\hline 1.00 & $1-1,99$ & 33 & 19 & 79 \\
\hline 1.10 & $1-1,109$ & 46 & 34 & 74 \\
\hline 1.19 & $1-1,118$ & 39 & 27 & 69 \\
\hline 1.29 & $1-1,128$ & 51 & & \\
\hline 1.42 & $1-1,141$ & 41 & 23 & 77 \\
\hline 1.52 & $1-2,1$ & 21 & 17 & 17 \\
\hline 1.61 & $1-2,10$ & 55 & 35 & 82 \\
\hline 1.71 & $1-2,20$ & 70 & 30 & 86 \\
\hline 1.79 & $1-2,28$ & 67 & 38 & 78 \\
\hline 1.90 & $1-2,39$ & 82 & 43 & 87 \\
\hline 2.01 & $1-2,50$ & 77 & & \\
\hline 2.15 & $1-2,64$ & 81 & 46 & 92 \\
\hline 2.19 & $1-2,68$ & 83 & & \\
\hline 2.30 & $1-2,79$ & 61 & 25 & 79 \\
\hline 2.41 & $1-2,90$ & 25 & 27 & 85 \\
\hline 2.49 & $1-2,98$ & 28 & 18 & 53 \\
\hline 2.60 & $1-2,109$ & 42 & 18 & 91 \\
\hline 2.71 & $1-2,120$ & 35 & 15 & 6 \\
\hline 2.80 & $1-2,129$ & 23 & 14 & 68 \\
\hline 2.95 & $1-2,144$ & 45 & 32 & 71 \\
\hline 3.02 & $1-3,1$ & 64 & 45 & 82 \\
\hline 3.10 & $1-3,9$ & 42 & 42 & 71 \\
\hline 3.20 & $1-3,19$ & 50 & 41 & 77 \\
\hline 3.31 & $1-3,30$ & 62 & 50 & 81 \\
\hline 3.45 & $1-3,44$ & 70 & & \\
\hline 3.50 & $1-3,49$ & 68 & 34 & 84 \\
\hline 3.59 & $1-3,58$ & 63 & 38 & 80 \\
\hline 3.70 & $1-3,69$ & 41 & 25 & 80 \\
\hline 3.79 & $1-3,78$ & 65 & 37 & 86 \\
\hline 3.89 & $1-3,88$ & 72 & 39 & 92 \\
\hline 3.99 & $1, \mathrm{CC}(4)$ & 50 & 25 & 69 \\
\hline 4.05 & $1, C C(10)$ & 50 & 21 & 65 \\
\hline 4.29 & $2-1,28$ & 34 & 22 & 66 \\
\hline 4.41 & $2-1,40$ & 44 & 25 & 76 \\
\hline 4.50 & $2-1,49$ & 29 & 17 & 57 \\
\hline 4.60 & $2-1,59$ & 33 & 24 & 65 \\
\hline 4.70 & $2-1,69$ & 44 & 22 & 71 \\
\hline 4.81 & $2-1,80$ & 77 & 32 & 86 \\
\hline 4.91 & $2-1,90$ & 71 & 38 & 86 \\
\hline 4.99 & $2-1,98$ & 60 & & \\
\hline 5.11 & $2-1,110$ & 75 & 34 & 87 \\
\hline 5.20 & $2-1,119$ & 68 & 31 & 88 \\
\hline 5.31 & $2-1,130$ & 71 & 33 & 89 \\
\hline 5.40 & $2-1,139$ & 66 & 37 & 85 \\
\hline 5.52 & $2-2,1$ & 84 & 44 & 93 \\
\hline 5.61 & $2-2,10$ & 81 & & \\
\hline 5.72 & $2-2,21$ & 44 & 23 & 74 \\
\hline 5.76 & $2-2,25$ & 52 & 28 & 69 \\
\hline 5.81 & $2-2,30$ & 53 & 54 & 60 \\
\hline 5.90 & $2-2,39$ & 72 & 24 & 92 \\
\hline 6.00 & $2-2,49$ & 78 & 26 & 86 \\
\hline 6.10 & $2-2,59$ & 72 & 36 & 93 \\
\hline 6.15 & $2-2,64$ & 79 & 35 & 96 \\
\hline 6.21 & $2-2,70$ & 88 & 12 & 98 \\
\hline 6.26 & $2-2,75$ & 91 & 16 & 100 \\
\hline 6.32 & $2-2,81$ & 91 & 16 & 98 \\
\hline 6.41 & $2-2,90$ & 91 & 19 & 97 \\
\hline 6.50 & $2-2,99$ & 87 & & \\
\hline 6.61 & $2-2,110$ & 87 & 15 & 90 \\
\hline
\end{tabular}

Table 1. (Continued).

\begin{tabular}{|c|c|c|c|c|}
\hline Depth (m) & $\begin{array}{l}\text { Core-Section } \\
\text { (level in } \mathrm{cm} \text { ) }\end{array}$ & $\mathrm{CaCO}_{3}(\%)$ & CF $(\%)$ & $\begin{array}{c}\mathrm{CF} \\
\mathrm{CaCO}_{3}(\%)\end{array}$ \\
\hline 6.71 & $2-2,120$ & 83 & 21 & 88 \\
\hline 6.81 & $2-2,130$ & 46 & 15 & 80 \\
\hline 6.91 & $2-2,140$ & 46 & 13 & \\
\hline 7.02 & $2-3,1$ & 32 & 22 & 33 \\
\hline 7.11 & $2-3,10$ & 15 & 21 & \\
\hline 7.20 & $2-3,19$ & 12 & 12 & 29 \\
\hline 7.31 & $2-3,30$ & 23 & 29 & 48 \\
\hline 7.39 & $2-3,38$ & 34 & & \\
\hline 7.51 & $2-3,50$ & 29 & 15 & 60 \\
\hline 7.61 & $2-3,60$ & 34 & 24 & 57 \\
\hline 7.69 & $2-3,68$ & 42 & 19 & 71 \\
\hline 7.79 & $2-3,78$ & 78 & 22 & 48 \\
\hline 7.92 & $2-3,91$ & 44 & 28 & 69 \\
\hline 8.01 & $2-3,100$ & 42 & 20 & 69 \\
\hline 8.09 & $2-3,108$ & 44 & 19 & 72 \\
\hline 8.21 & $2-3,120$ & 44 & 22 & 65 \\
\hline 8.30 & $2-3,129$ & 45 & 16 & 60 \\
\hline 8.40 & $2-3,139$ & 33 & 10 & 64 \\
\hline 8.52 & $2-4,1$ & 67 & 19 & 87 \\
\hline 8.55 & $2-4,4$ & 88 & 16 & 83 \\
\hline 8.61 & $2-4,10$ & 76 & 10 & 80 \\
\hline 8.68 & $2-4,17$ & 49 & 5 & 65 \\
\hline 8.75 & $2-4,24$ & 81 & & \\
\hline 8.81 & $2-4,30$ & 88 & 14 & 81 \\
\hline 8.89 & $2-4,38$ & 88 & 16 & 91 \\
\hline 8.98 & $2, \mathrm{CC}(8)$ & 90 & 36 & 95 \\
\hline 9.02 & $3-1,1$ & 69 & 22 & 85 \\
\hline 9.11 & $3-1,10$ & 49 & 14 & 73 \\
\hline 9.21 & $3-1,20$ & 66 & 20 & 75 \\
\hline 9.31 & $3-1,30$ & 66 & 21 & 85 \\
\hline 9.41 & $3-1,40$ & 27 & 11 & 67 \\
\hline 9.48 & $3-1,47$ & 76 & 13 & 76 \\
\hline 9.58 & $3-1,57$ & 82 & 13 & 78 \\
\hline 9.69 & $3-1,68$ & 64 & 24 & 76 \\
\hline 9.79 & $3-1,78$ & 42 & 27 & 74 \\
\hline 9.87 & $3-1,86$ & 77 & 46 & 92 \\
\hline 9.98 & $3-1,97$ & 79 & 35 & 86 \\
\hline 10.06 & $3-1,105$ & 71 & 28 & 80 \\
\hline 10.18 & $3-1,117$ & 79 & 50 & 87 \\
\hline 10.30 & $3-1,129$ & 80 & 54 & 88 \\
\hline 10.37 & $3-1,136$ & 85 & & \\
\hline 10.55 & $3-2,4$ & 79 & 43 & 87 \\
\hline 10.68 & $3-2,17$ & 64 & 41 & 76 \\
\hline 10.75 & $3-2,24$ & 31 & 17 & 56 \\
\hline 10.84 & $3-2,33$ & 25 & 21 & 41 \\
\hline 10.88 & $3-2,37$ & 25 & 14 & 66 \\
\hline 10.98 & $3-2,47$ & 49 & 35 & 85 \\
\hline 11.14 & $3-2,63$ & 14 & 24 & 59 \\
\hline 11.24 & $3-2,73$ & 23 & 21 & 47 \\
\hline 11.34 & $3-2,83$ & 34 & 23 & 67 \\
\hline 11.39 & $3-2,88$ & 24 & & \\
\hline 11.44 & $3-2,93$ & 22 & 23 & 50 \\
\hline 11.50 & $3-2,99$ & 15 & 12 & 67 \\
\hline 11.64 & $3-2,113$ & 26 & 13 & 60 \\
\hline 11.73 & $3-2,122$ & 40 & 21 & 72 \\
\hline 11.84 & $3-2,133$ & 23 & 10 & 56 \\
\hline 11.97 & $3-2,146$ & 33 & 16 & 67 \\
\hline 12.04 & $3-3,3$ & 28 & 24 & 73 \\
\hline 12.13 & $3-3,12$ & 31 & 19 & 57 \\
\hline 12.20 & $3-3,19$ & 3 (ash) & 15 & 10 \\
\hline 12.33 & $3-3,32$ & 45 & 21 & 60 \\
\hline 12.45 & $3-3,44$ & 29 & 15 & 76 \\
\hline 12.50 & $3-3,49$ & 83 & 54 & 96 \\
\hline 12.58 & $3-3,57$ & 89 & 37 & 92 \\
\hline 12.64 & $3-3,63$ & 84 & 36 & 95 \\
\hline 12.75 & $3-3,74$ & 77 & 34 & 91 \\
\hline 12.85 & $3-3,84$ & 66 & 29 & 77 \\
\hline 12.94 & $3-3,93$ & 43 & 16 & 70 \\
\hline 12.98 & $3-3,97$ & 42 & & \\
\hline
\end{tabular}


Table 1. (Continued).

\begin{tabular}{|c|c|c|c|c|}
\hline Depth (m) & $\begin{array}{l}\text { Core-Section } \\
\text { (level in } \mathrm{cm} \text { ) }\end{array}$ & $\mathrm{CaCO}_{3}(\%)$ & CF $(\%)$ & $\begin{array}{c}\mathrm{CF} \\
\mathrm{CaCO}_{3}(\%)\end{array}$ \\
\hline 13.15 & $3-3,114$ & 4 & 6 & 17 \\
\hline 13.19 & $3-3,118$ & 8 & & \\
\hline 13.22 & $3-3,121$ & 10 & 9 & 23 \\
\hline 13.30 & $3-3,129$ & 17 & 15 & 67 \\
\hline 13.40 & $3-3,139$ & 40 & 28 & 87 \\
\hline 13.47 & $3-3,146$ & 57 & & \\
\hline 13.54 & $3-4,3$ & 71 & 46 & 91 \\
\hline 13.63 & $3-4,12$ & 62 & 35 & 82 \\
\hline 13.75 & $3-4,24$ & 49 & 27 & 90 \\
\hline 13.78 & $3-4,27$ & 18 & 19 & 55 \\
\hline 13.95 & $3, \mathrm{CC}(15)$ & 18 & 16 & 50 \\
\hline 14.02 & $4-1,1$ & 48 & 24 & 80 \\
\hline 14.11 & $4-1,10$ & 52 & 31 & 82 \\
\hline 14.21 & $4-1,20$ & 70 & 16 & 60 \\
\hline 14.31 & $4-1,30$ & 59 & 22 & 74 \\
\hline 14.42 & $4-1,41$ & 18 & 21 & 27 \\
\hline 14.52 & $4-1,51$ & 14 & 6 & 38 \\
\hline 14.60 & $4-1,59$ & 73 & 27 & 53 \\
\hline 14.72 & $4-1,71$ & 61 & 32 & 65 \\
\hline 14.80 & $4-1,79$ & 64 & 43 & 83 \\
\hline 14.90 & $4-1,89$ & 79 & 41 & 86 \\
\hline 15.12 & $4-1,111$ & 80 & 33 & 88 \\
\hline 15.20 & $4-1,119$ & 67 & 35 & 83 \\
\hline 15.30 & $4-1,129$ & 76 & & \\
\hline 15.40 & $4-1,139$ & 75 & 43 & 85 \\
\hline 15.52 & $4-2,1$ & 78 & 41 & 86 \\
\hline 15.62 & $4-2,11$ & 77 & 27 & \\
\hline 15.70 & $4-2,19$ & 41 & 24 & 49 \\
\hline 15.79 & $4-2,28$ & 37 & 28 & 49 \\
\hline 15.92 & $4-2,41$ & 33 & & \\
\hline 16.01 & $4-2,50$ & 21 & 16 & 48 \\
\hline 16.10 & $4-2,59$ & 8 & 18 & 34 \\
\hline 16.19 & $4-2,68$ & 7 & 13 & 12 \\
\hline 16.30 & $4-2,79$ & 9 & 3 & 8 \\
\hline 16.41 & $4-2,90$ & 10 & 4 & $50(?)$ \\
\hline 16.51 & $4-2,100$ & 19 & & \\
\hline 16.62 & $4-2,111$ & 60 & 39 & 85 \\
\hline 16.71 & $4-2,120$ & 60 & 40 & 86 \\
\hline 16.78 & $4-2,127$ & 60 & 33 & 80 \\
\hline 16.89 & $4-2,138$ & 36 & 11 & 59 \\
\hline 16.96 & $4-2,145$ & 16 & 5 & 23 \\
\hline 17.02 & $4-3,1$ & 18 & 19 & 44 \\
\hline 17.10 & $4-3,9$ & 4 & 24 & 37 \\
\hline 17.20 & $4-3,19$ & 50 & 25 & 75 \\
\hline 17.30 & $4-3,29$ & 49 & & \\
\hline 17.39 & $4-3,38$ & 64 & 50 & 68 \\
\hline 17.49 & $4-3,48$ & 88 & 40 & 89 \\
\hline 17.60 & $4-3,59$ & 55 & 28 & 82 \\
\hline 17.71 & $4-3,70$ & 10 & 20 & 17 \\
\hline 17.81 & $4-3,80$ & 70 & 39 & 89 \\
\hline 17.86 & $4-3,85$ & 79 & 50 & 84 \\
\hline 17.91 & $4-3,90$ & 62 & 31 & 79 \\
\hline 18.02 & $4-3,101$ & 47 & & \\
\hline 18.06 & $4-3,105$ & 49 & 25 & 80 \\
\hline 18.13 & $4-3,112$ & 18 & 10 & 45 \\
\hline 18.20 & $4-3,119$ & 3 & 6 & $33(?)$ \\
\hline 18.31 & $4-3,130$ & 60 & 41 & 86 \\
\hline 18.42 & $4-3,141$ & 69 & 38 & 88 \\
\hline 18.52 & $4-4,1$ & 52 & 35 & 87 \\
\hline 18.61 & $4-4,10$ & 28 & 10 & 56 \\
\hline 18.68 & $4-4,17$ & 11 & 7 & 38 \\
\hline 18.71 & $4-4,20$ & 34 & 18 & 77 \\
\hline 18.81 & $4-4,30$ & 53 & 31 & 84 \\
\hline 18.89 & $4, \mathrm{CC}(8)$ & 51 & 22 & 80 \\
\hline 18.98 & $4, \mathrm{CC}(17)$ & 28 & 19 & 72 \\
\hline 19.02 & $5-1,1$ & 63 & 40 & 87 \\
\hline 19.09 & $5-1,8$ & 37 & 16 & 71 \\
\hline 19.20 & $5-1,19$ & 56 & 33 & 77 \\
\hline 19.31 & $5-1,30$ & 74 & 61 & 82 \\
\hline
\end{tabular}

Table 1. (Continued).

\begin{tabular}{|c|c|c|c|c|}
\hline Depth (m) & $\begin{array}{l}\text { Core-Section } \\
\text { (level in } \mathrm{cm} \text { ) }\end{array}$ & $\mathrm{CaCO}_{3}(\%)$ & CF $(\%)$ & $\begin{array}{c}\mathrm{CF} \\
\mathrm{CaCO}_{3}(\%)\end{array}$ \\
\hline 19.40 & $5-1,39$ & 72 & 43 & 85 \\
\hline 19.56 & $5-1,55$ & 56 & 35 & 71 \\
\hline 19.61 & $5-1,60$ & 36 & 18 & 56 \\
\hline 19.72 & $5-1,71$ & 33 & 25 & 77 \\
\hline 19.80 & $5-1,79$ & 62 & 29 & 78 \\
\hline 19.91 & $5-1,90$ & 91 & 38 & 96 \\
\hline 19.97 & $5-1,96$ & 89 & 36 & 93 \\
\hline 20.12 & $5-1,111$ & 83 & 44 & 91 \\
\hline 20.20 & $5-1,119$ & 75 & 41 & 88 \\
\hline 20.30 & $5-1,129$ & 59 & 35 & 89 \\
\hline 20.43 & $5-1,142$ & 16 & 24 & 71 \\
\hline 20.52 & $5-2,1$ & 31 & 24 & 75 \\
\hline 20.59 & $5-2,8$ & 26 & 8 & 35 \\
\hline 20.70 & $5-2,19$ & 68 & 31 & 77 \\
\hline 20.81 & $5-2,30$ & 10 & 9 & 54 \\
\hline 20.92 & $5-2,41$ & 9 & 43 & 86 \\
\hline 21.03 & $5-2,52$ & 34 & 5 & 58 \\
\hline 21.11 & $5-2,60$ & 43 & 46 & 58 \\
\hline 21.23 & $5-2,72$ & 23 & 18 & 62 \\
\hline 21.32 & $5-2,81$ & 41 & 16 & 65 \\
\hline 21.41 & $5-2,90$ & 69 & 44 & 79 \\
\hline 21.49 & $5-2,98$ & 86 & 51 & 92 \\
\hline 21.58 & $5-2,107$ & 85 & 49 & 87 \\
\hline 21.71 & $5-2,120$ & 70 & 57 & 89 \\
\hline 21.82 & $5-2,131$ & 31 & 24 & 64 \\
\hline 21.93 & $5-2,142$ & 35 & & \\
\hline 22.02 & $5-3,1$ & 45 & 40 & 42 \\
\hline 22.10 & $5-3,9$ & 39 & 13 & 73 \\
\hline 22.20 & $5-3,19$ & 41 & 17 & 77 \\
\hline 22.32 & $5-3,31$ & 64 & 53 & 74 \\
\hline 22.43 & $5-3,42$ & 84 & 28 & 90 \\
\hline 22.53 & $5-3,52$ & 63 & 15 & 60 \\
\hline 22.61 & $5-3,60$ & 47 & 37 & 79 \\
\hline 22.69 & $5-3,68$ & 16 & 10 & 37 \\
\hline 22.81 & $5-3,80$ & 37 & 17 & 56 \\
\hline 22.91 & $5-3,90$ & 48 & 30 & 63 \\
\hline 23.01 & $5-3,100$ & 54 & 31 & 78 \\
\hline 23.11 & $5-3,110$ & 77 & 42 & 86 \\
\hline 23.20 & $5-3,119$ & 49 & 31 & 39 \\
\hline 23.31 & $5-3,130$ & 17 & 15 & 33 \\
\hline 23.43 & $5-3,142$ & 45 & 11 & 78 \\
\hline 23.52 & $5-4,1$ & 29 & 14 & 24 \\
\hline 23.63 & $5-4,12$ & 4 & 5 & 0 \\
\hline 22.67 & $5-4,16$ & 12 & & \\
\hline 23.71 & $5-4,20$ & 19 & 6 & 9 \\
\hline 23.81 & $5-4,30$ & 72 & 42 & 80 \\
\hline 23.91 & $5-4,40$ & 85 & 41 & 89 \\
\hline 23.95 & $5, \mathrm{CC}(4)$ & 84 & 39 & 92 \\
\hline 24.03 & $5, \mathrm{CC}(12)$ & 36 & 17 & 25 \\
\hline 29.08 & $7-1,7$ & 54 & 36 & 57 \\
\hline 29.19 & $7-1,18$ & 56 & & \\
\hline 29.29 & $7-1,28$ & 82 & 51 & 85 \\
\hline 29.39 & $7-1,38$ & 72 & 37 & 89 \\
\hline 29.49 & $7-1,48$ & 78 & 44 & 94 \\
\hline 29.61 & $7-1,60$ & 89 & 39 & 95 \\
\hline 29.72 & $7-1,71$ & 84 & 30 & 96 \\
\hline 29.82 & $7-1,81$ & 79 & 36 & 87 \\
\hline 29.90 & $7-1,89$ & 52 & 39 & 75 \\
\hline 29.99 & $7-1,98$ & 53 & 30 & 85 \\
\hline 30.09 & $7-1,108$ & 83 & 39 & 93 \\
\hline 30.21 & $7-1,120$ & 83 & 54 & 92 \\
\hline 30.32 & $7-1,131$ & 72 & 43 & 77 \\
\hline 30.42 & $7-1,141$ & 54 & 28 & 62 \\
\hline 30.52 & $7-2,1$ & 15 & 33 & 0 \\
\hline 30.61 & $7-2,10$ & 30 & 17 & 47 \\
\hline 30.72 & $7-2,21$ & 28 & 13 & 57 \\
\hline 30.81 & $7-2,30$ & 15 & 13 & 2 \\
\hline 30.92 & $7-2,41$ & 59 & 28 & 84 \\
\hline 31.01 & $7-2,50$ & 17 & 12 & 35 \\
\hline
\end{tabular}


Table 1. (Continued).

\begin{tabular}{|c|c|c|c|c|}
\hline Depth (m) & $\begin{array}{l}\text { Core-Section } \\
\text { (level in cm) }\end{array}$ & $\mathrm{CaCO}_{3}(\%)$ & CF $(\%)$ & $\begin{array}{c}\mathrm{CF} \\
\mathrm{CaCO}_{3}(\%)\end{array}$ \\
\hline 31.10 & $7-2,59$ & 54 & 30 & 86 \\
\hline 31.19 & $7-2,68$ & 84 & 29 & 90 \\
\hline 31.28 & $7-2,77$ & 84 & 44 & 88 \\
\hline 31.39 & $7-2,88$ & 46 & 40 & 69 \\
\hline 31.54 & $7-2,103$ & 33 & 21 & 74 \\
\hline 31.59 & $7-2,108$ & 7 & 12 & 47 \\
\hline 31.70 & $7-2,119$ & 85 & 49 & 97 \\
\hline 31.82 & $7-2,131$ & 87 & 40 & 96 \\
\hline 31.88 & $7-2,137$ & 89 & & \\
\hline 31.96 & $7-2,145$ & 85 & 37 & 92 \\
\hline 32.02 & $7-3,1$ & 84 & 46 & 91 \\
\hline 32.12 & $7-3,11$ & 66 & 25 & 83 \\
\hline 32.22 & $7-3,21$ & 52 & 17 & 50 \\
\hline 32.31 & $7-3,30$ & 13 & 9 & 0 \\
\hline 32.38 & $7-3,37$ & 46 & 17 & \\
\hline 32.54 & $7-3,53$ & 40 & 14 & 72 \\
\hline 32.59 & $7-3,58$ & 81 & 37 & 86 \\
\hline 32.68 & $7-3,67$ & 77 & 32 & 85 \\
\hline 32.83 & $7-3,82$ & 77 & 29 & 87 \\
\hline 32.87 & $7-3,86$ & 58 & 16 & 76 \\
\hline 32.99 & $7-3,98$ & 24 & 9 & 41 \\
\hline 33.12 & $7-3,111$ & 85 & 39 & 92 \\
\hline 33.20 & $7-3,119$ & 89 & 44 & 96 \\
\hline 33.32 & $7-3,131$ & 90 & 50 & 96 \\
\hline 33.46 & $7-3,145$ & 89 & & \\
\hline 33.52 & $7-4,1$ & 89 & 40 & 98 \\
\hline 33.57 & $7-4,6$ & 88 & 29 & 95 \\
\hline 33.67 & $7, \mathrm{CC}(6)$ & 89 & 37 & 96 \\
\hline 33.77 & $7, \mathrm{CC}(16)$ & 88 & 39 & 95 \\
\hline 34.03 & $8-1,2$ & 60 & 33 & 69 \\
\hline 34.09 & $8-1,8$ & 18 & 4 & 7 \\
\hline 34.19 & $8-1,18$ & 68 & 32 & 75 \\
\hline 34.31 & $8-1,30$ & 66 & 25 & 80 \\
\hline 34.43 & $8-1,42$ & 66 & 22 & 84 \\
\hline 34.50 & $8-1,49$ & 71 & 29 & \\
\hline 34.58 & $8-1,57$ & 72 & 36 & 78 \\
\hline 34.69 & $8-1,68$ & 83 & 39 & 86 \\
\hline 34.79 & $8-1,78$ & 80 & 39 & 89 \\
\hline 34.88 & $8-1,87$ & 81 & 39 & 90 \\
\hline 34.99 & $8-1,98$ & 61 & & \\
\hline 35.11 & $8-1,111$ & 73 & 24 & 85 \\
\hline 35.20 & $8-1,119$ & 63 & 24 & 81 \\
\hline 35.32 & $8-1,131$ & 71 & 25 & 86 \\
\hline 35.46 & $8-1,145$ & 55 & 23 & 89 \\
\hline 35.52 & $8-2,1$ & 79 & 38 & 98 \\
\hline 35.61 & $8-2,10$ & 88 & 33 & 96 \\
\hline 35.68 & $8-2,17$ & 92 & & \\
\hline 35.81 & $8-2,30$ & 90 & 37 & 98 \\
\hline 35.92 & $8-2,41$ & 88 & 33 & 98 \\
\hline 36.01 & $8-2,50$ & 89 & 28 & 98 \\
\hline 36.11 & $8-2,60$ & 90 & 36 & 100 \\
\hline 36.21 & $8-2,70$ & 87 & 29 & 96 \\
\hline 36.31 & $8-2,80$ & 87 & 27 & 97 \\
\hline 36.41 & $8-2,90$ & 87 & 31 & 97 \\
\hline 36.52 & $8-2,101$ & 86 & 21 & 94 \\
\hline 36.61 & $8-2,110$ & 87 & 26 & 97 \\
\hline 36.66 & $8-2,115$ & 89 & 34 & 96 \\
\hline 36.81 & $8-2,130$ & 84 & 14 & 94 \\
\hline 36.96 & $8-2,145$ & 53 & 14 & 77 \\
\hline 37.02 & $8-3,1$ & 55 & 31 & 63 \\
\hline 37.09 & $8-3,8$ & 53 & 14 & 67 \\
\hline 37.18 & $8-3,17$ & 29 & & \\
\hline 37.31 & $8-3,30$ & 61 & 26 & 76 \\
\hline 37.41 & $8-3,40$ & 58 & 15 & 72 \\
\hline 37.53 & $8-3,52$ & 72 & & \\
\hline 37.61 & $8-3,60$ & 79 & 26 & 84 \\
\hline 37.72 & $8-3,71$ & 49 & 12 & 72 \\
\hline 37.79 & $8-3,78$ & 68 & 16 & 87 \\
\hline 37.87 & $8-3,86$ & 86 & 33 & 89 \\
\hline
\end{tabular}

Table 1. (Continued).

\begin{tabular}{|c|c|c|c|c|}
\hline Depth (m) & $\begin{array}{l}\text { Core-Section } \\
\text { (level in } \mathrm{cm} \text { ) }\end{array}$ & $\mathrm{CaCO}_{3}(\%)$ & CF $(\%)$ & $\begin{array}{c}\mathrm{CF} \\
\mathrm{CaCO}_{3}(\%)\end{array}$ \\
\hline 37.95 & $8-3,94$ & 79 & 13 & 90 \\
\hline 38.01 & $8-3,100$ & 68 & 20 & 78 \\
\hline 38.13 & $8-3,112$ & 38 & 14 & 49 \\
\hline 38.21 & $8-3,120$ & 6 & 31 & 4 \\
\hline 38.29 & $8-3,128$ & 29 & 11 & 50 \\
\hline 38.42 & $8-3,141$ & 74 & 42 & 93 \\
\hline 38.52 & $8-4,1$ & 90 & 36 & $?$ \\
\hline 38.61 & $8-4,10$ & 79 & 33 & 90 \\
\hline 38.71 & $8-4,20$ & 85 & 34 & 93 \\
\hline 38.81 & $8-4,30$ & 78 & 47 & 89 \\
\hline 38.90 & $8-4,39$ & 83 & 34 & 91 \\
\hline 38.97 & $8, C C(6)$ & 31 & 12 & 54 \\
\hline 39.06 & $9-1,5$ & 77 & 37 & 88 \\
\hline 39.16 & $9-1,15$ & 73 & 31 & 88 \\
\hline 39.34 & $9-1,33$ & 85 & 26 & 92 \\
\hline 39.48 & $9-1,47$ & 81 & 19 & 94 \\
\hline 39.65 & $9-1,64$ & 46 & 20 & 47 \\
\hline 39.75 & $9-1,74$ & 33 & 26 & 86 \\
\hline 39.95 & $9-1,94$ & 84 & 46 & 85 \\
\hline 40.00 & $9-1,99$ & 78 & & \\
\hline 40.07 & $9-1,106$ & 58 & 30 & 77 \\
\hline 40.17 & $9-1,116$ & 87 & 41 & 92 \\
\hline 40.28 & $9-1,127$ & 80 & 29 & 89 \\
\hline 40.40 & $9-1,139$ & 56 & 22 & 71 \\
\hline 40.48 & $9-1,147$ & 86 & 35 & 100 \\
\hline 40.56 & $9-2,5$ & 85 & 45 & 92 \\
\hline 40.66 & $9-2,15$ & 81 & 36 & 87 \\
\hline 40.78 & $9-2,27$ & 68 & 32 & 71 \\
\hline 40.90 & $9-2,39$ & 35 & 20 & 46 \\
\hline 40.96 & $9-2,45$ & 29 & 35 & 40 \\
\hline 41.07 & $9-2,56$ & 80 & 40 & 83 \\
\hline 41.18 & $9-2,67$ & 84 & 37 & 85 \\
\hline 41.28 & $9-2,77$ & 64 & 21 & 70 \\
\hline 41.35 & $9-2,84$ & 24 & 14 & 40 \\
\hline 41.38 & $9-2,87$ & 62 & 44 & 60 \\
\hline 41.47 & $9-2,96$ & 82 & & \\
\hline 41.55 & $9-2,104$ & 81 & 17 & 92 \\
\hline 41.61 & $9-2,110$ & 82 & 22 & 90 \\
\hline 41.69 & $9-2,118$ & 55 & 23 & 62 \\
\hline 41.79 & $9-2,128$ & 15 & 6 & 58 \\
\hline 41.86 & $9-2,135$ & 52 & 22 & 58 \\
\hline 41.98 & $9-2,147$ & 23 & & \\
\hline 41.99 & $9-2,148$ & 21 & 22 & 30 \\
\hline 42.06 & $9-3,5$ & 27 & 19 & 24 \\
\hline 42.14 & $9-3,13$ & 44 & 26 & 73 \\
\hline 42.18 & $9-3,17$ & 92 & & \\
\hline 42.28 & $9-3,27$ & 90 & 25 & 97 \\
\hline 42.39 & $9-3,38$ & 92 & 28 & 100 \\
\hline 42.49 & $9-3,48$ & 92 & 36 & 100 \\
\hline 42.58 & $9-3,57$ & 90 & 34 & 98 \\
\hline 42.69 & $9-3,68$ & 90 & 34 & 100 \\
\hline 42.79 & $9-3,78$ & 93 & 24 & 98 \\
\hline 42.95 & $9-3,94$ & 90 & 20 & 97 \\
\hline 43.07 & $9-3,106$ & 90 & 26 & 100 \\
\hline 43.16 & $9-3,115$ & 90 & 27 & 91 \\
\hline 43.18 & $9-3,117$ & 67 & 27 & 94 \\
\hline 43.28 & $9-3,127$ & 88 & 19 & 98 \\
\hline 43.40 & $9-3,139$ & 78 & 14 & 85 \\
\hline 43.50 & $9-3,149$ & 60 & 20 & 64 \\
\hline 43.56 & $9-4,5$ & 78 & 50 & 92 \\
\hline 43.66 & $9-4,15$ & 89 & 26 & 93 \\
\hline 43.77 & $9-4,26$ & 87 & 37 & 88 \\
\hline 43.92 & $9, \mathrm{CC}(13)$ & 86 & 31 & 92 \\
\hline 44.02 & $10-1,1$ & 83 & 35 & 90 \\
\hline 44.12 & $10-1,11$ & 85 & 24 & 68 \\
\hline 44.26 & $10-1,25$ & 93 & 23 & 97 \\
\hline 44.32 & $10-1,31$ & 91 & 32 & 93 \\
\hline 44.43 & $10-1,42$ & 92 & 30 & 97 \\
\hline 44.53 & $10-1,52$ & 89 & 17 & 100 \\
\hline
\end{tabular}


Table 1. (Continued).

\begin{tabular}{|c|c|c|c|c|}
\hline Depth (m) & $\begin{array}{l}\text { Core-Section } \\
\text { (level in } \mathrm{cm} \text { ) }\end{array}$ & $\mathrm{CaCO}_{3}(\%)$ & CF $(\%)$ & $\begin{array}{c}\mathrm{CF} \\
\mathrm{CaCO}_{3}(\%)\end{array}$ \\
\hline 44.61 & $10-1,60$ & 90 & 11 & \\
\hline 44.73 & $10-1,72$ & 92 & 16 & 100 \\
\hline 44.83 & $10-1,82$ & 94 & 22 & 97 \\
\hline 44.88 & $10-1,87$ & 92 & & \\
\hline 44.93 & $10-1,92$ & 89 & 21 & 98 \\
\hline 45.04 & $10-1,103$ & 91 & 19 & 100 \\
\hline 45.08 & $10-1,107$ & 92 & & \\
\hline 45.23 & $10-1,122$ & 91 & 28 & 96 \\
\hline 45.30 & $10-1,129$ & 92 & 25 & 97 \\
\hline 45.49 & $10-1,148$ & 92 & 21 & 98 \\
\hline 45.54 & $10-2,3$ & 89 & 34 & 93 \\
\hline 45.63 & $10-2,12$ & 90 & 34 & 95 \\
\hline 45.72 & $10-2,21$ & 91 & 46 & 91 \\
\hline 45.82 & $10-2,31$ & 83 & 36 & 93 \\
\hline 45.96 & $10-2,45$ & 87 & 45 & 92 \\
\hline 46.03 & $10-2,52$ & 90 & 25 & 96 \\
\hline 46.13 & $10-2,62$ & 91 & 34 & 95 \\
\hline 46.23 & $10-2,72$ & 92 & 39 & 94 \\
\hline 46.34 & $10-2,83$ & 92 & 32 & 96 \\
\hline 46.42 & $10-2,91$ & 89 & 22 & 97 \\
\hline 46.53 & $10-2,102$ & 89 & 24 & 100 \\
\hline 46.58 & $10-2,107$ & 90 & & \\
\hline 46.64 & $10-2,113$ & 92 & 27 & 98 \\
\hline 46.72 & $10-2,121$ & 93 & 32 & 96 \\
\hline 46.80 & $10-2,129$ & 91 & 24 & 98 \\
\hline 46.88 & $10-2,137$ & 92 & & \\
\hline 46.96 & $10-2,145$ & 92 & 28 & 100 \\
\hline 47.04 & $10-3,3$ & 89 & 26 & 99 \\
\hline 47.12 & $10-3,11$ & 92 & 19 & 98 \\
\hline 47.22 & $10-3,21$ & 91 & 15 & 100 \\
\hline 47.31 & $10-3,30$ & 88 & 18 & 97 \\
\hline 47.43 & $10-3,42$ & 92 & 15 & 100 \\
\hline 47.55 & $10-3,54$ & 93 & 18 & 100 \\
\hline 47.63 & $10-3,62$ & 93 & 27 & 99 \\
\hline 47.73 & $10-3,72$ & 92 & 23 & 100 \\
\hline 47.85 & $10-3,84$ & 94 & 22 & 100 \\
\hline 47.93 & $10-3,92$ & 92 & 30 & 99 \\
\hline 48.06 & $10-3,105$ & 93 & 23 & 100 \\
\hline 48.13 & $10-3,112$ & 92 & 26 & 98 \\
\hline 48.23 & $10-3,122$ & 93 & 40 & 98 \\
\hline 43.25 & $10-3,134$ & 92 & 30 & 98 \\
\hline 48.47 & $10-3,146$ & 92 & & \\
\hline 48.54 & $10-4,3$ & 92 & 31 & 98 \\
\hline 48.67 & $10-4,16$ & 93 & 19 & 85 \\
\hline 48.76 & $10-4,25$ & 90 & 19 & 100 \\
\hline 48.78 & $10-4,27$ & 87 & 23 & 95 \\
\hline 48.90 & $10-4,39$ & 94 & 19 & 98 \\
\hline 49.01 & $10, \mathrm{CC}(8)$ & 90 & 18 & 100 \\
\hline 49.07 & $11-1,6$ & 83 & 27 & 79 \\
\hline 49.21 & $11-1,20$ & 90 & 19 & 98 \\
\hline 49.29 & $11-1,28$ & 92 & 25 & 100 \\
\hline 49.41 & $11-1,40$ & 90 & 15 & 100 \\
\hline 49.51 & $11-1,50$ & 89 & 22 & 98 \\
\hline 49.59 & $11-1,58$ & 91 & 25 & 98 \\
\hline 49.71 & $11-1,70$ & 84 & 21 & 84 \\
\hline 49.80 & $11-1,79$ & 89 & 19 & 100 \\
\hline 49.88 & $11-1,87$ & 92 & 20 & 98 \\
\hline 50.00 & $11-1,99$ & 92 & 16 & 100 \\
\hline 50.11 & $11-1,110$ & 93 & 16 & 100 \\
\hline 50.18 & $11-1,117$ & 93 & 22 & 100 \\
\hline 50.31 & $11-1,130$ & 93 & 18 & 97 \\
\hline 50.41 & $11-1,140$ & 92 & 21 & 93 \\
\hline 50.55 & $11-2,4$ & 93 & 26 & 97 \\
\hline 50.64 & $11-2,13$ & 91 & 14 & 97 \\
\hline 50.70 & $11-2,19$ & 91 & 15 & 94 \\
\hline 50.85 & $11-2,34$ & 91 & 18 & 88 \\
\hline 50.91 & $11-2,40$ & 92 & 14 & 97 \\
\hline 50.98 & $11-2,47$ & 91 & & \\
\hline 51.09 & $11-2,58$ & 89 & 25 & 99 \\
\hline 51.24 & $11-2,73$ & 90 & 12 & 93 \\
\hline
\end{tabular}

Table 1. (Continued).

\begin{tabular}{|c|c|c|c|c|}
\hline Depth (m) & $\begin{array}{l}\text { Core-Section } \\
\text { (level in } \mathrm{cm} \text { ) }\end{array}$ & $\mathrm{CaCO}_{3}(\%)$ & CF $(\%)$ & $\begin{array}{c}\mathrm{CF} \\
\mathrm{CaCO}_{3}(\%)\end{array}$ \\
\hline 51.43 & $11-2,92$ & 92 & 15 & 95 \\
\hline 51.53 & $11-2,102$ & 93 & 10 & 96 \\
\hline 51.66 & $11-2,115$ & 94 & 7 & 95 \\
\hline 51.68 & $11-2,117$ & 93 & 9 & 96 \\
\hline 51.79 & $11-2,128$ & 91 & 11 & 96 \\
\hline 51.89 & $11-2,138$ & 92 & 16 & 96 \\
\hline 52.05 & $11-3,4$ & 91 & 17 & 94 \\
\hline 52.14 & $11-3,13$ & 91 & 15 & 97 \\
\hline 52.24 & $11-3,23$ & 92 & 15 & 100 \\
\hline 52.33 & $11-3,32$ & 92 & 26 & 99 \\
\hline 52.38 & $11-3,37$ & 93 & & \\
\hline 52.48 & $11-3,47$ & 93 & & \\
\hline 52.58 & $11-3,57$ & 94 & 26 & 94 \\
\hline 52.75 & $11-3,74$ & 95 & 15 & 100 \\
\hline 52.87 & $11-3,86$ & 94 & 17 & 96 \\
\hline 52.98 & $11-3,97$ & 93 & & \\
\hline 53.09 & $11-3,108$ & 95 & 10 & 96 \\
\hline 53.23 & $11-3,122$ & 93 & 9 & 97 \\
\hline 53.35 & $11-3,134$ & 92 & 6 & 93 \\
\hline 53.55 & $11-4,4$ & 92 & 8 & 96 \\
\hline 53.67 & $11-4,16$ & 93 & 8 & 100 \\
\hline 53.78 & $11-4,27$ & 92 & 11 & 97 \\
\hline 53.90 & $11-4,39$ & 95 & 7 & 88 \\
\hline 54.02 & $11, \mathrm{CC}(8)$ & 94 & 11 & 94 \\
\hline 54.07 & $12-1,6$ & 88 & 17 & 76 \\
\hline 54.20 & $12-1,19$ & 93 & 14 & 84 \\
\hline 54.33 & $12-1,32$ & 95 & 13 & 95 \\
\hline 54.40 & $12-1,39$ & 92 & 7 & 88 \\
\hline 54.55 & $12-1,54$ & 93 & 7 & 91 \\
\hline 54.63 & $12-1,62$ & 92 & 10 & 93 \\
\hline 54.75 & $12-1,74$ & 94 & 6 & 100 \\
\hline 54.85 & $12-1,84$ & 94 & 9 & 96 \\
\hline 54.91 & $12-1,90$ & 94 & 12 & 96 \\
\hline 55.08 & $12-1,107$ & 94 & 13 & 100 \\
\hline 55.23 & $12-1,122$ & 91 & 13 & 98 \\
\hline 55.36 & $12-1,135$ & 90 & 11 & 100 \\
\hline 55.49 & $12-1,148$ & 91 & 12 & 100 \\
\hline 55.55 & $12-2,4$ & 93 & 15 & 98 \\
\hline 55.66 & $12-2,15$ & 92 & 13 & 100 \\
\hline 55.75 & $12-2,24$ & 93 & 12 & 100 \\
\hline 55.83 & $12-2,32$ & 93 & 21 & 95 \\
\hline 55.95 & $12-2,44$ & 93 & 11 & 100 \\
\hline 56.08 & $12-2,57$ & 92 & 19 & 95 \\
\hline 56.19 & $12-2,68$ & 93 & 18 & 98 \\
\hline 56.29 & $12-2,78$ & 94 & 17 & 100 \\
\hline 56.41 & $12-2,90$ & 93 & 18 & 97 \\
\hline 56.53 & $12-2,102$ & 92 & 16 & 97 \\
\hline 56.68 & $12-2,117$ & 91 & 24 & 95 \\
\hline 56.79 & $12-2,128$ & 91 & 9 & 100 \\
\hline 56.95 & $12-2,144$ & 90 & 8 & 100 \\
\hline 57.04 & $12-3,3$ & 87 & 11 & 88 \\
\hline 57.08 & $12-3,8$ & 88 & & \\
\hline 57.17 & $12-3,16$ & 89 & 11 & 100 \\
\hline 57.33 & $12-3,32$ & 94 & 11 & 95 \\
\hline 57.46 & $12-3,45$ & 93 & 10 & 97 \\
\hline 57.59 & $12-3,58$ & 94 & 13 & 96 \\
\hline 57.75 & $12-3,74$ & 93 & 14 & 100 \\
\hline 57.78 & $12-3,77$ & 94 & & \\
\hline 57.91 & $12-3,90$ & 90 & 9 & 93 \\
\hline 57.98 & $12-3,97$ & 91 & 7 & 100 \\
\hline 58.16 & $12-3,115$ & 89 & 9 & 100 \\
\hline 58.19 & $12-3,118$ & 87 & 9 & 100 \\
\hline 58.30 & $12-3,129$ & 92 & 9 & 100 \\
\hline 58.39 & $12-3,138$ & 91 & 9 & 100 \\
\hline 58.54 & $12-4,3$ & 95 & 9 & 100 \\
\hline 58.66 & $12-4,15$ & 87 & & \\
\hline 58.74 & $12-4,23$ & 93 & 7 & 100 \\
\hline 58.78 & $12-4,27$ & 95 & 9 & 100 \\
\hline 58.86 & $12, \mathrm{CC}(5)$ & 91 & 11 & 100 \\
\hline 58.96 & $12, \mathrm{CC}(15)$ & 92 & 10 & 96 \\
\hline
\end{tabular}




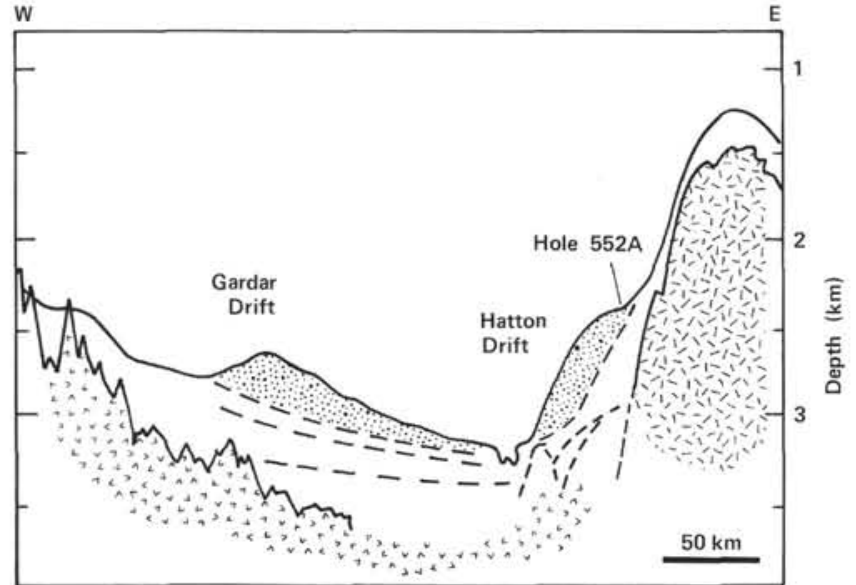

Figure 2. Position of Hole 552A on the western flank of Rockall Plateau. Sediments of the Gardar and Hatton drifts are stippled. Subbottom information generalized from Site 552 chapter (this volume).

the carbonate content is controlled by glacial-interglacial depositional regimes (Ruddiman and McIntyre, 1976); coccoliths and foraminifers are deposited two to three times more rapidly during interglacials (a function of surface water productivity), whereas noncalcareous terrigenous sediments are deposited more rapidly under glacial climatic conditions as a result of ice-rafting.

\section{Biogenous Constituents}

The calcareous oozes occur in shades of white and light gray and are somewhat mottled by bioturbation. Laminated structures are rare, although an occasional thin layer $(<1 \mathrm{~cm}$ thick) of foraminiferal "sand" may result from winnowing by bottom currents. The carbonate content of the interglacial calcareous oozes fluctuates between 70 and $90 \%$, attaining a consistent value of $95 \%$ in the underlying late Pliocene preglacial oozes below $44 \mathrm{~m}$ (Fig. 3).

Carbonate content is clearly correlated with the coarse fraction $(>74 \mu \mathrm{m})$ content. Even a cursory examination of the sediment reveals that the largest proportion of the coarse fraction of the interglacial stages consists of foraminiferal tests (Fig. 3). Except for the low-carbonate spikes during glacial episodes, most of the coarse fraction is of high carbonate content. It is interesting to note that during interglacial stages the coarse fraction components (foraminifers) form 40 to $60 \%$ of the total sediment, whereas in the preglacial late Pliocene oozes, the coarse fraction makes up generally less than $20 \%$ of the sediment.

The interplay between these three curves-carbonate percent, coarse fraction percent, and carbonate percent of the coarse fraction (Fig. 3)-reflects the variation of the calcareous components produced under the rapidly changing climatic regimes of the past $2.4 \mathrm{~m}$.y. The low carbonate-low productivity segments of the carbonate curve most probably reflect the prevalence of sea ice and downwelling within the broadly cyclonic circulation of surface water in the Rockall-Greenland sector of the glacial North Atlantic. Under interglacial conditions the return of the warm North Atlantic Drift stimulates an increase in biologic productivity. The spikes of low coarse fraction carbonate associated with the glacial stages suggest particularly intense cold periods during which seaice cover may have been so extensive that surface productivity in this area was effectively eliminated for periods of time long enough to be recorded in this section.

There is also a positive correlation between biogenic silica and total carbonate content (Baldauf, this volume). Biosiliceous productivity thus corresponds to that of the calcareous microfossils. Biogenic silica, however, only becomes abundant where carbonate content exceeds $90 \%$. Sponge spicules occur commonly in sediments with carbonate values as low as $80 \%$; the more delicate diatoms and radiolarians, however, only occur in sediments of higher carbonate content. The correspondence of diatom and calcareous microfossil abundance suggests that planktonic productivity of both is controlled by similar climatically induced environmental factors in the surface ocean.

A particularly distinct interval of biosiliceous dissolution, occurring between 8 and $33 \mathrm{~m}$ sub-bottom (0.441.8 m.y., Fig. 9; Baldauf, this volume), does not allow a complete correlation of siliceous/calcareous planktonic productivity. Although occasional samples within this interval contain well-preserved diatom flora, the majority of samples are barren of diatoms. This barren interval has probably resulted from a complex of environmental factors affecting productivity and preservation. An interval which may be similar was recorded by Schrader and Fenner (1976) in the Norwegian Sea (DSDP Leg 38). At these sites, the barren interval was also punctuated by occasional samples with an abundant assemblage of temperate diatoms. It is suggested that these occurrences of abundant diatom content represent isolated episodes of warmth during which the North Atlantic Drift is able to penetrate into the Norwegian Sea.

Organic carbon content (Kaltenback et al., this volume) also has a cyclical variability. Interglacial carbonate oozes have organic carbon contents of $0.06-0.08 \%$, whereas in the glacial muds the organic carbon values are in the $0.14-0.20 \%$ range (Fig. 4). Analysis by RockEval pyrolysis also reveals that organic carbon content of glacial sediments contains a higher proportion of residual or reworked carbon (commonly termed "highly oxidized, residual nonsource," Fig. 5). The higher levels of organic carbon, therefore, reflect an influx of reworked organic matter during glacial climates, undoubtedly a result of lower sea levels and increased erosion of the continents and continental shelves.

Sediments enriched in organic phosphorus often characterize organic material of pelagic origin. Interglacial organic material of these sediments is enriched in phosphorus $(\mathrm{c} / \mathrm{p}<25)$, whereas in glacial sediments organic phosphorus was not detected. Relatively high levels of organic phosphorus also characterize the late Pliocene calcareous oozes deposited prior to the inception of glaciation. These high values of organic phosphorus considered together with the low values of residual carbon suggest a predominately marine origin for the organic material of the interglacial sediments. 

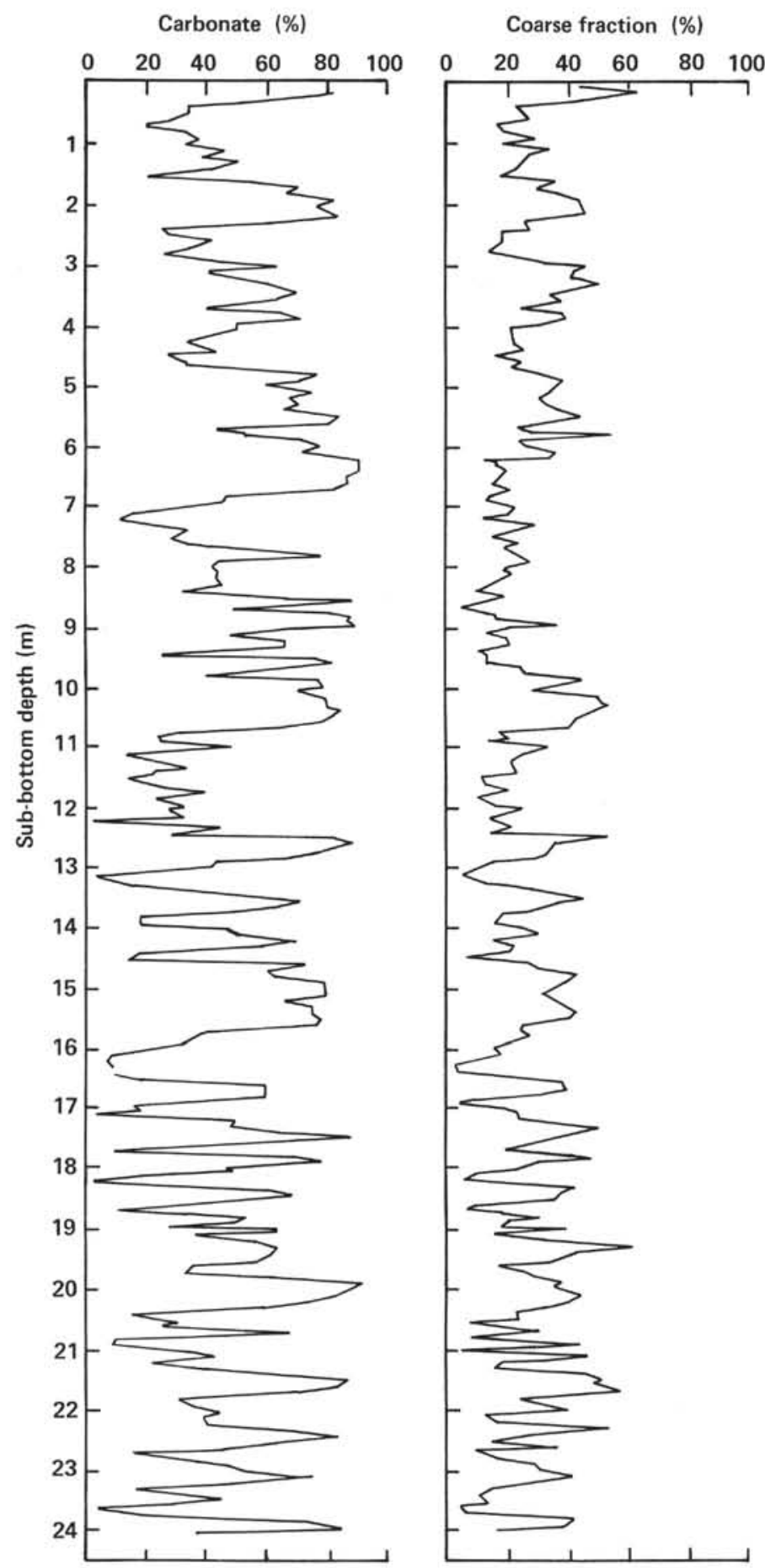

Carbonate of coarse fraction (\%)

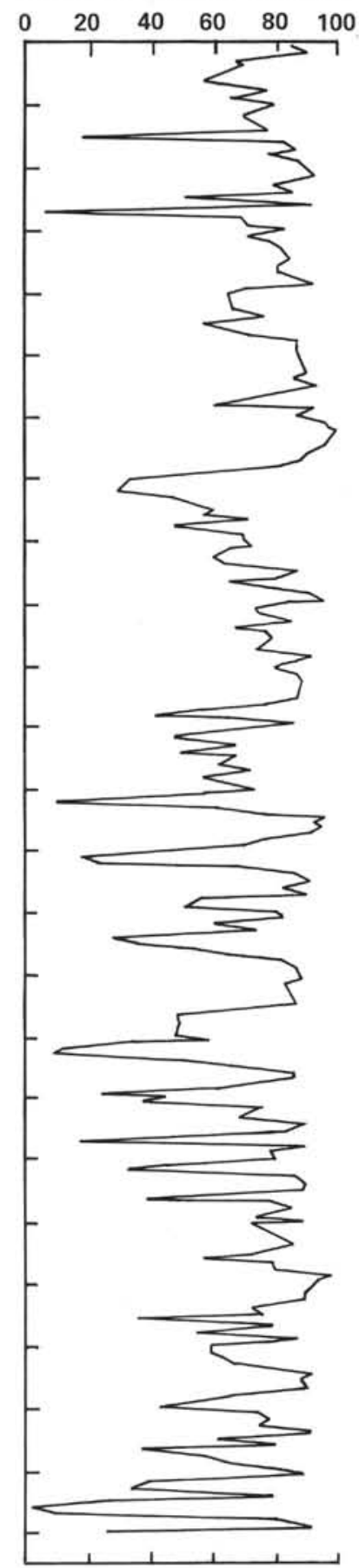

Figure 3. Percent carbonate, coarse fraction $(>74 \mu \mathrm{m})$, and coarse fraction carbonate plotted on a depth scale, Hole 552A, Cores 1-5 and Cores 7-12.

Because of the difficulty in measuring such small amounts of organic material, samples from only two climatic cycles were examined. We note that, with respect to organic matter, there is apparently little difference between a glacial-interglacial cycle occurring early or late in the Plio-Pleistocene sequence.

\section{Terrigenous Components}

The bulk of the noncarbonate fraction of these sediments is concentrated in the glacial sequences. XRD analy- sis indicates that the clay composition is almost totally smectite in the interglacial oozes, whereas the glacial muds contain the predominately terrigenous clays-illite, kaolinite, and chlorite. This observation reinforces the suggestion that sediments of the glacial stages originate primarily from continental sources related to lower sea levels and increased rates of continental erosion.

The terrigenous coarse fraction is arkosic in composition (Morton, this volume); the presence of feldspar with polycrystalline and strained monocrystalline quartz and 

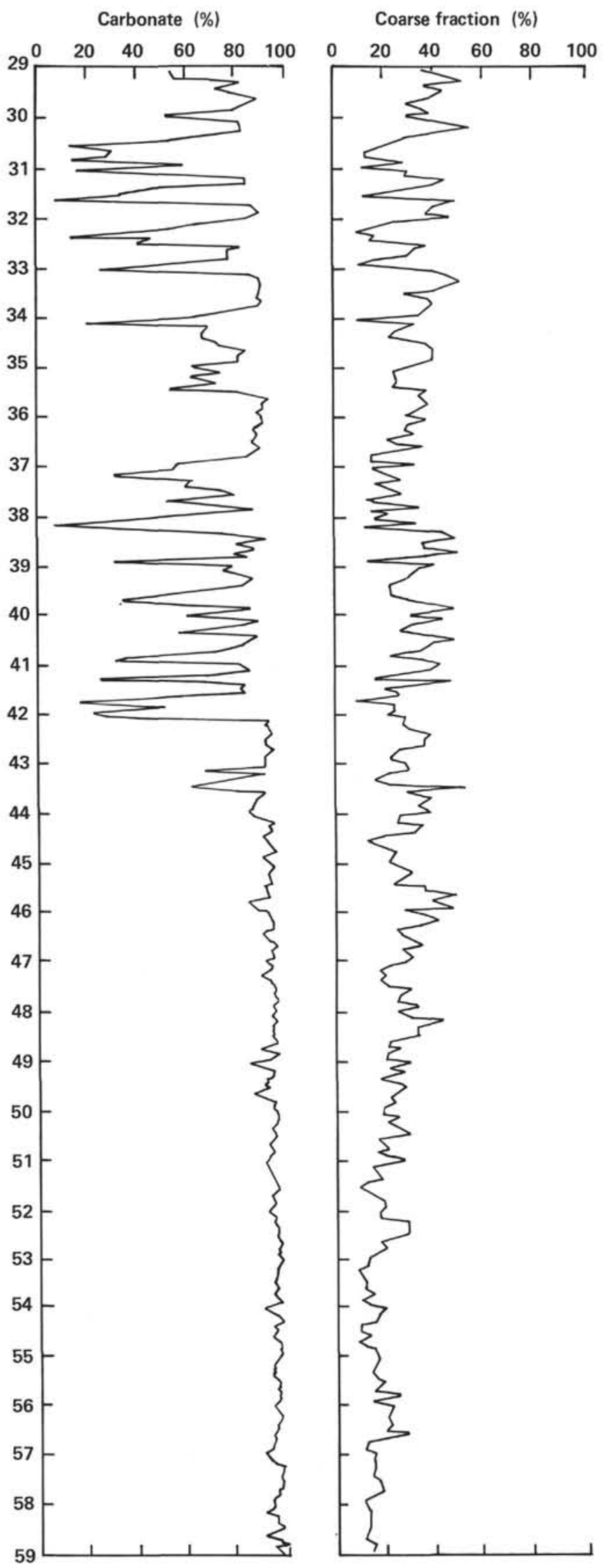

Carbonate of coarse fraction (\%)

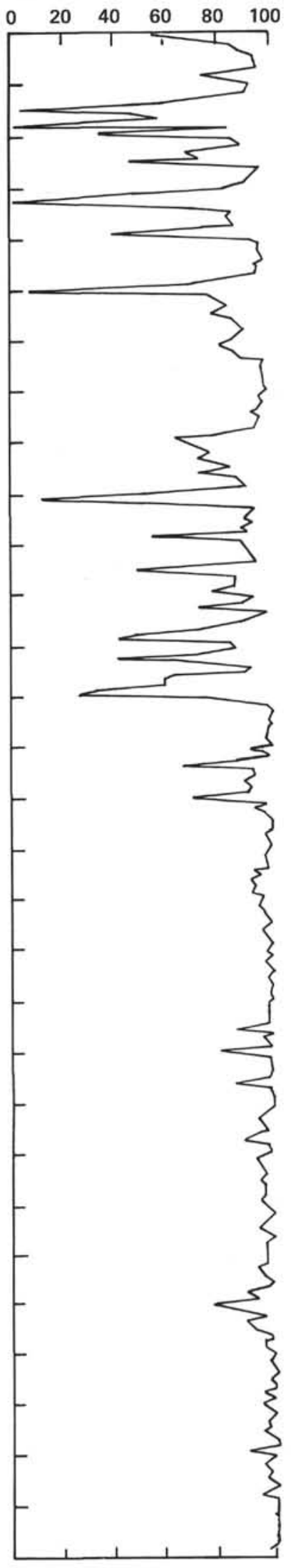

Figure 3. (Continued). 


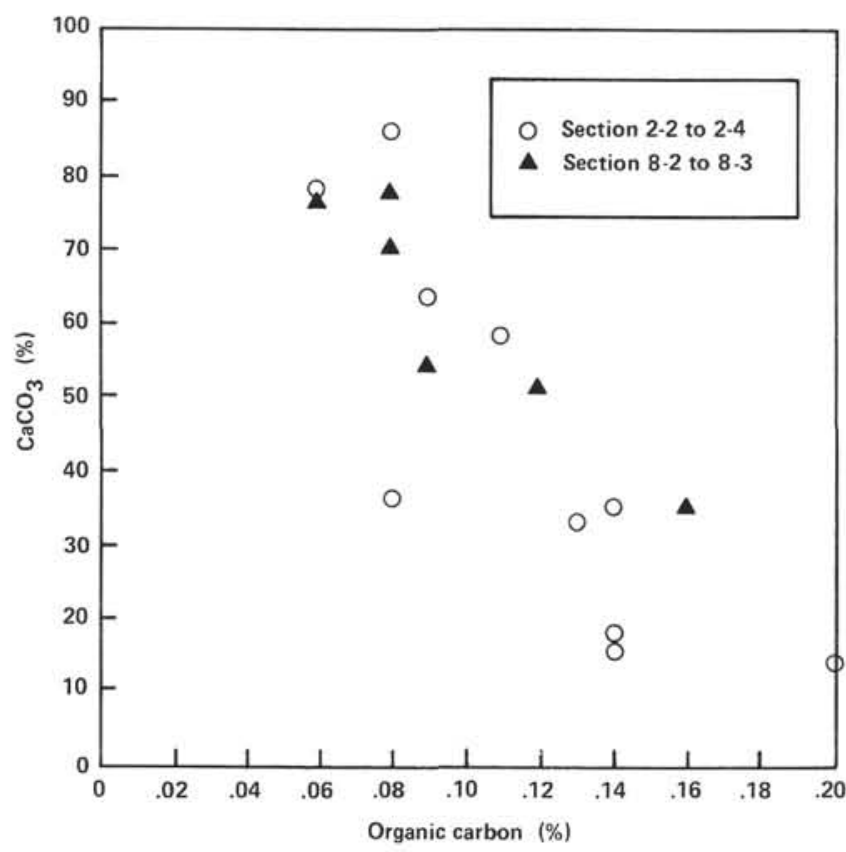

Figure 4. Percent calcium carbonate plotted against organic carbon for two climatic cycles (Hole 552A, Cores 2 and 8).

metamorphic rock fragments suggests a source largely of metamorphic basement. The occurrence of basalt grains also indicates some contribution from nearby exposed lavas. Heavy mineral constituents similarly suggest a mixed metamorphic-basaltic terrane (Morton, this volume). The most immediate single source for these sediment grains is Greenland, which satisfies the criteria of possessing both extensive tracts of metamorphic basement and large areas of exposed basalt. The sheltered position of Site 552 on the upper reaches of the Hatton Drift serves to eliminate other potential source areas to the east. Given the presence of scattered large pebbles (Site 552 chapter), ice-rafting is the most likely process of sediment transport for these sands and silts.

Pyroclastic input is sporadic; it is a consistent but relatively minor constituent in the lower part of the sequence (Cores 5 to 9), disappears in Cores 3 and 4, and is present again in Cores 1 and 2 (Morton, this volume). Most of the pyroclastic material is glassy, varying between slightly to highly vesiculated; both clear and brown types occur in variable proportions. Minor components of pyroclastic origin include augite, plagioclase, and opaque grains. These constituents of volcanic ash are similar to those of DSDP Site 404 (Harrison et al., 1979) and also to ashes of Plio-Pleistocene age at DSDP Site 346 on the Icelandic Plateau (Sylvester, 1976).

It is generally accepted that the volcanic ash of PlioPleistocene age in the North Atlantic and NorwegianGreenland Sea originated from eruptions on Iceland (Ruddiman and Glover, 1972; Sylvester, 1976; Harrison et al, 1979; Donn and Ninkovitch, 1980). The explosive nature of the volcanism is ascribed to the interaction of the magma with water, in this case with glacial meltwater, in the manner described by Heiken (1975).

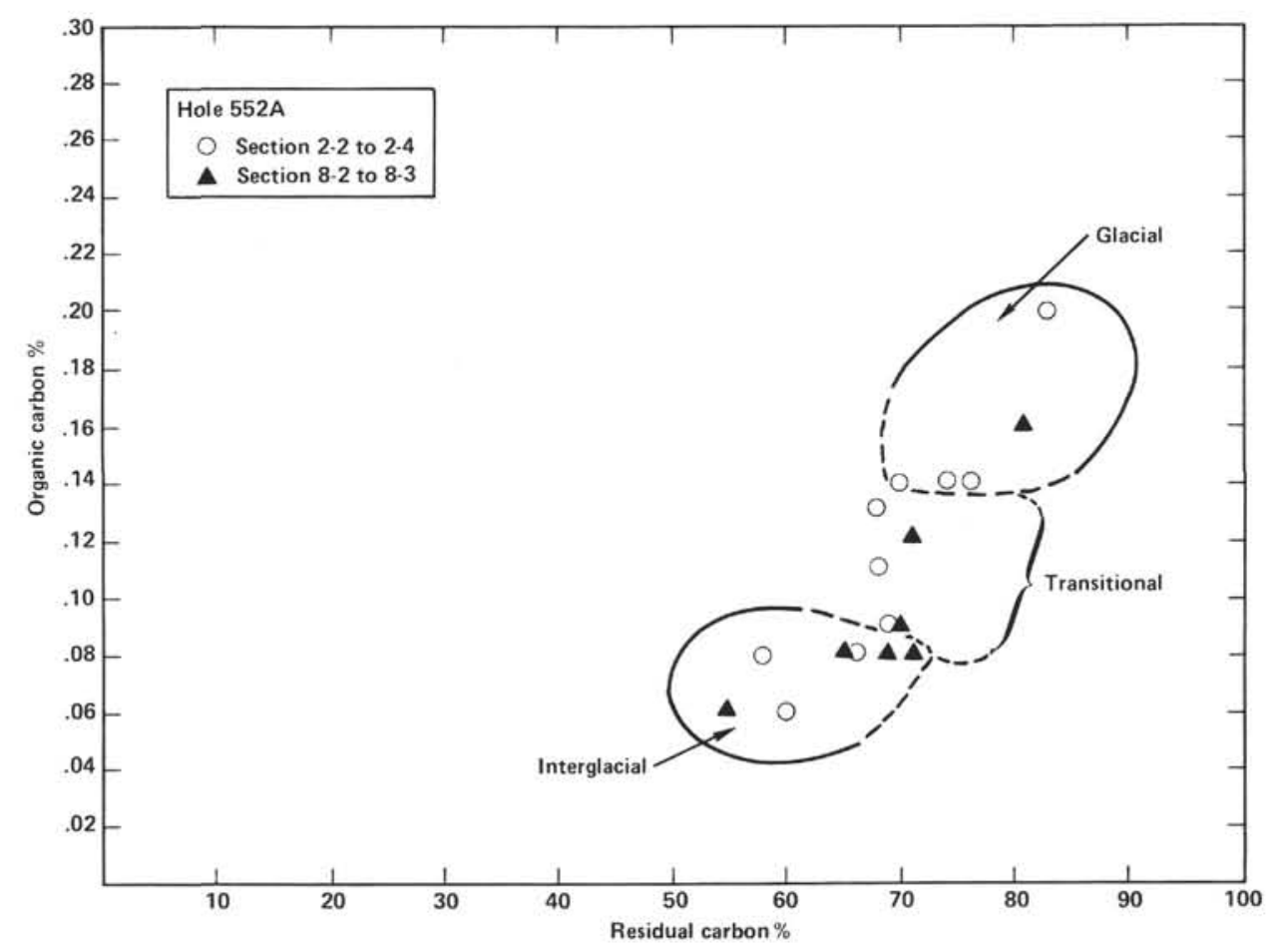

Figure 5. Percent organic carbon plotted against residual carbon for two climatic cycles (Hole 552A, Cores 2 and 8 ). 


\section{STABLE ISOTOPE STRATIGRAPHY}

\section{Oxygen Isotope Record: Hole 552A, Cores 1-5}

In Figures 6 and 7A, the oxygen isotope, paleomagnetic, and carbonate data for the upper five cores are shown together with the oxygen isotope record of piston core V28-239 (Shackleton and Opdyke, 1976). We have used V28-239 here because, although it is known to include changes in accumulation rate, it contains an almost complete record of Plio-Pleistocene climatic cycles. A comparison of DSDP Hole 552A with V28-238, which records an almost uniform accumulation rate, may be found in Shackleton et al. (1984). Correlation of the isotopic stage boundaries reveals marked variations in accumulation rate in DSDP Hole 552A, a not unexpected result considering the lithological variations that are evident.

We have erected isotopic stage boundaries for Hole $552 \mathrm{~A}$ by comparisons of the curves and paleomagnetic control points. For this part of the section, the global oxygen isotopic record is relatively well known. Hole $552 \mathrm{~A}$ is clearly correlative with the Pacific record and complete to the extent of preserving every isotope stage. Shackleton and Opdyke $(1973 ; 1976)$ recognized 23 isotopic stages in the late and middle Pleistocene. Through comparison of Hole 552A with the Pacific records, we have extended this numbering system to at least interglacial stage 25 , which is defined as including the base of the Jaramillo subchron at 0.98 m.y. ago.

\section{Late Pliocene Onset of Ice-rafting}

The climatic record is less well known below the isotopic stages established for the middle and late Pleistocene. In Figure 8, the oxygen isotope data for the lower part of the sequence in Hole 552A is compared with the oxygen isotope data from piston core V28-179 (Shackleton and Opdyke, 1977), which covers a benthic oxygen isotope record back to $3.5 \mathrm{~m}$.y. To facilitate comparison, the plotting scale for both records has been adjusted at the indicated magnetostratigraphic boundaries; an approximate age scale is thus obtained.

Comparison between these two records is good considering the low accumulation rate, bioturbation, and relatively coarse sampling interval in V28-179. The amplitude of variation, however, is substantially greater in
Hole 552A than in V28-179. Since the accumulation rate of V28-179 was only about $0.55 \mathrm{~cm} / \mathrm{k}$.y., climatic extremes lasting only a few thousand years should be severely degraded by bioturbation.

Nannofossil extinction levels have been determined quantitatively for both cores and their ages estimated (Backman and Shackleton, 1983). Using these ages, the average accumulation rate over the pre-ice-rafting interval in Hole $552 \mathrm{~A}$ is estimated at approximately $1.7 \mathrm{~cm} /$ k.y. This is surprisingly close to the average for the upper Pleistocene $(1.8 \mathrm{~cm} / \mathrm{k} . \mathrm{y}$.), although the rate has certainly varied over the intense climatic fluctuations represented by the alternating oozes and muds.

Paleomagnetic and nannofossil stratigraphies of the sequence in Hole 552A give identical estimates for the age of the first major northern hemisphere glacial event2.37 m.y. Back to about $2.4 \mathrm{~m}$.y. there is a remarkable correspondence between the carbonate and oxygen isotope records of Hole 552A (Fig. 7B). It is evident that both parameters are causally related to glaciation. Moreover, the coincidence of the first isotopic event with the dramatic influx of ice-rafted debris at $2.4 \mathrm{~m}$.y. is clear evidence that this late Pliocene episode is related to a northern hemisphere occurrence, rather than to an Antarctic climatic event.

Comparison of the isotopic composition of benthic foraminifers at this level of the first glacial maximum with values in glacial stages in the upper part of the sequence show that the initial event represents a truly glacial interval with an ice volume similar to maxima of the middle and late Pleistocene. Although the section in Hole 552A may preserve a significant record of deepwater temperature variability in addition to the ice volume signal, the glacial event at 2.4 m.y. must certainly have been more severe than was recognized by Shackleton and Opdyke (1977). Almost a million years prior to the Pliocene/Pleistocene boundary (Backman et al., 1983), this glacial occurrence must represent the major environmental event of the late Neogene of the northern hemisphere.

Ice-rafted debris is absent prior to $2.4 \mathrm{~m} . \mathrm{y}$. This age for the initiation of major ice-rafting in the North Atlantic is considerably later than 3.0-3.2 m.y. determined by Berggren (1972) and Poore (1981). Their observations, however, were made on cores recovered by conventional

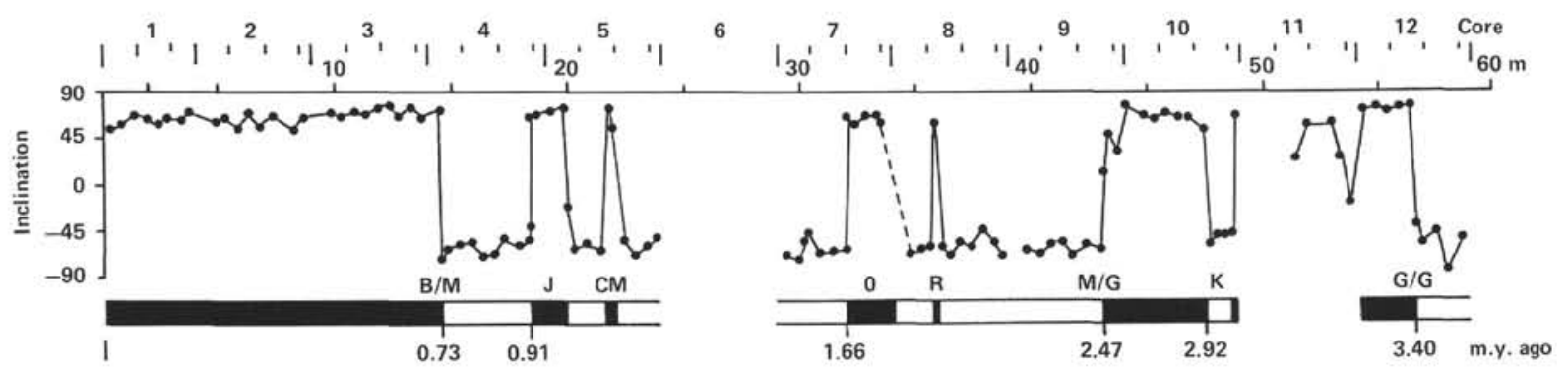

Figure 6. Magnetic record for Hole 552A, Cores 1-12. Demagnetized inclinations are shown only for apparently undisturbed parts of the cores. Time scale from Berggren et al., in press. $(B=$ Brunhes; $M=$ Matuyama; $\mathbf{J}=$ Jaramillo; $C M=C o b b$ Mountain; $O$ $=$ Olduvai; $\mathrm{R}=$ Reunion; $\mathrm{K}=$ Kaena; $\mathrm{G} / \mathrm{G}=$ Gauss $/$ Gilbert.) 

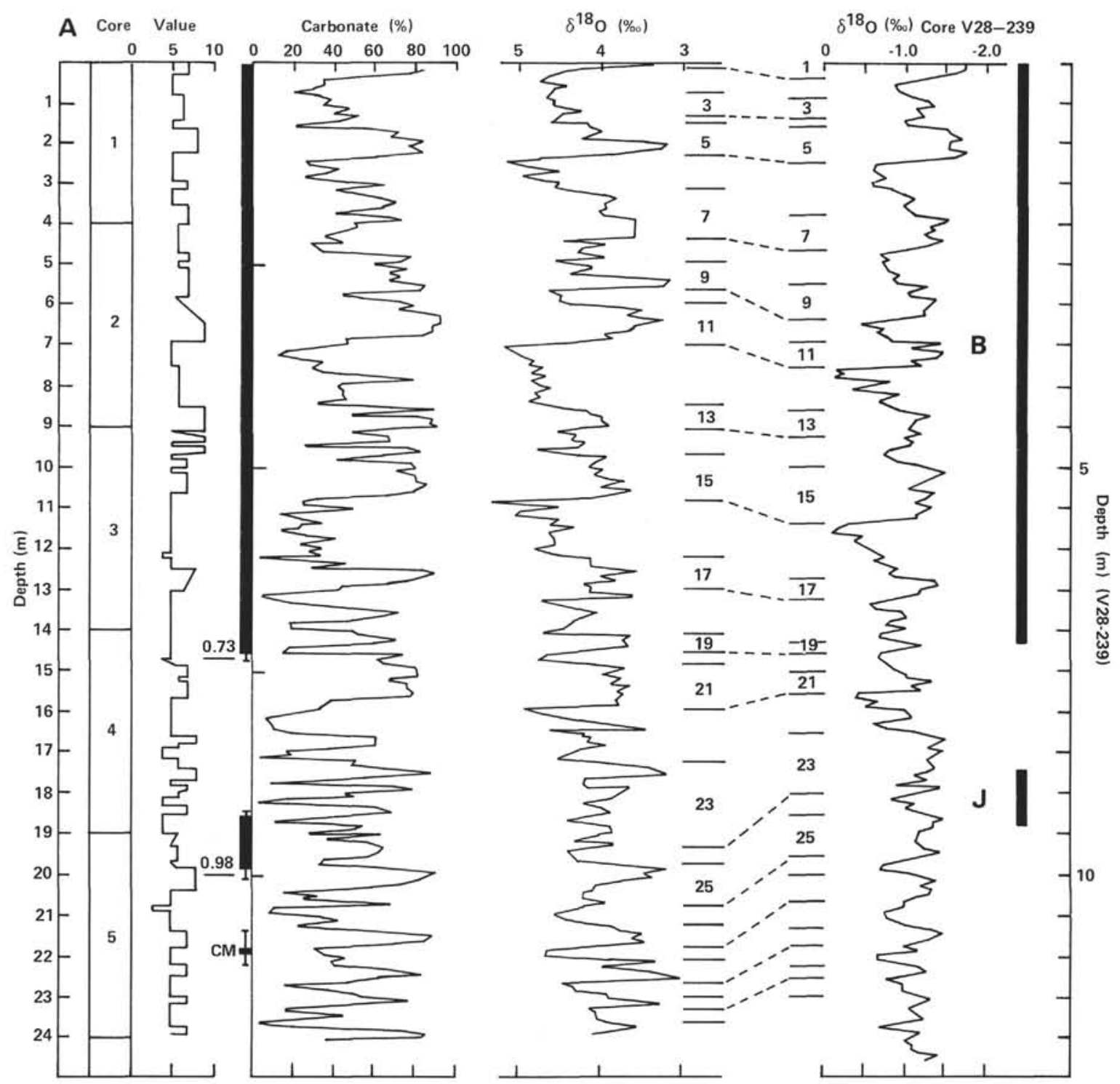

Figure 7. Summary of percent carbonate and oxygen isotope data for Hole 552A correlated with oxygen isotope record of V28239 (Shackleton and Opdyke, 1976). Records of both cores are plotted linearly with depth. Proposed oxygen isotope stage boundaries, paleomagnetic data, and color value (light [10]-dark [1]) are also indicated. A. Cores 1-5. B. Cores 7-12.

rotary drilling in which the sediments were greatly disturbed. Backman (1979) has re-dated the inception of icerafted sediments at DSDP Sites 111 and 116 at 2.5 m.y. ago. Prell (1982) and Hodell et al. (1983) presented evidence of isotopic variability around 3.2 m.y. ago; Prell interpreted this as representing a brief excursion in ice volume. The record preceding the initial glacial event at Hole 552A indicates that although there was considerable climatic variability somewhere, such glaciation as may have occurred prior to $2.5 \mathrm{~m}$.y. ago did not give rise to ice-rafting in the northeastern Atlantic.

\section{Carbon Isotope Record}

A water mass forming at the surface has the ${ }^{13} \mathrm{C}$ content of surface water and a high content of dissolved oxygen. During its passage through the deep reaches of the ocean, the water mass "ages"; dissolved oxygen is utilized by the oxidation of particulate organic matter sink- ing from the sea surface, and dissolved $\mathrm{CO}_{2}$ becomes isotopically lighter as a consequence of the addition of this isotopically light carbon (Kroopnick, 1980; Shackleton and Hall, this volume).

Carbon isotope records of Hole 552A and piston core V28-179 are compared in Figure 9. The data are plotted on the same isotopic scale, and the same species-dependent correction factors have been applied (Shackleton et al., 1984). In today's ocean there is an observed difference of a little over $1 \%$ between the two areas, reflecting the fact that the deep ocean is ventilated (younger water mass) in the North Atlantic. It is clear from the persistent offset in values that the water mass bathing Hatton Drift was younger than that of the deep Pacific throughout the interval studied. Moreover, since the high-frequency variability was approximately the same prior to the major climatic event at 2.4 m.y. ago as after, the ${ }^{13} \mathrm{C}$ variability was not forced by climatic cyclicity. 


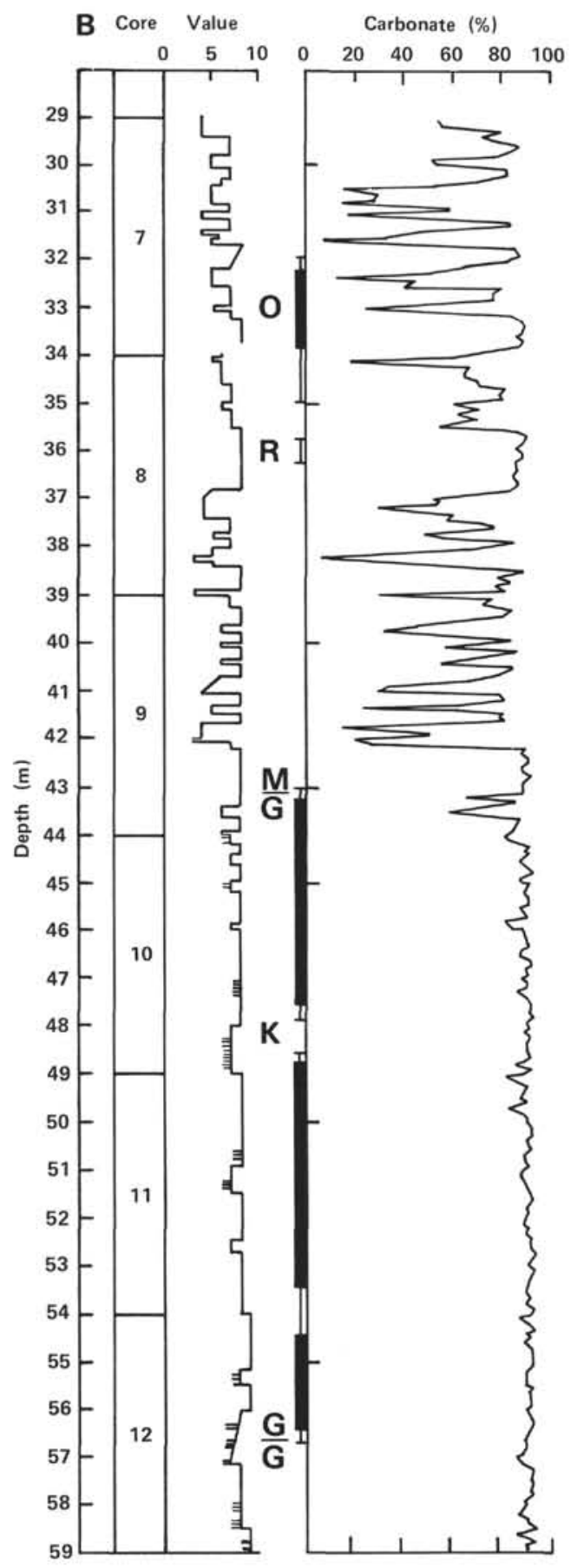

Figure 7. (Continued).

\section{CONCLUSIONS}

DSDP Hole 552A was cored with the HPC on Hatton Drift and obtained an almost complete and undisturbed sediment section spanning the late Neogene and Quaternary. Lithologic, faunal, isotopic, and paleomagnetic analyses indicate that the section represents the most complete deep sea record of climatic evolution hitherto recovered in the high latitude northern hemisphere. On the basis of the foregoing studies, the following conclusions seem warranted:

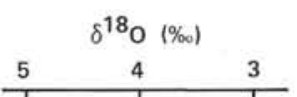

$\delta^{18} \mathrm{O}(\%)$ Core V28-239

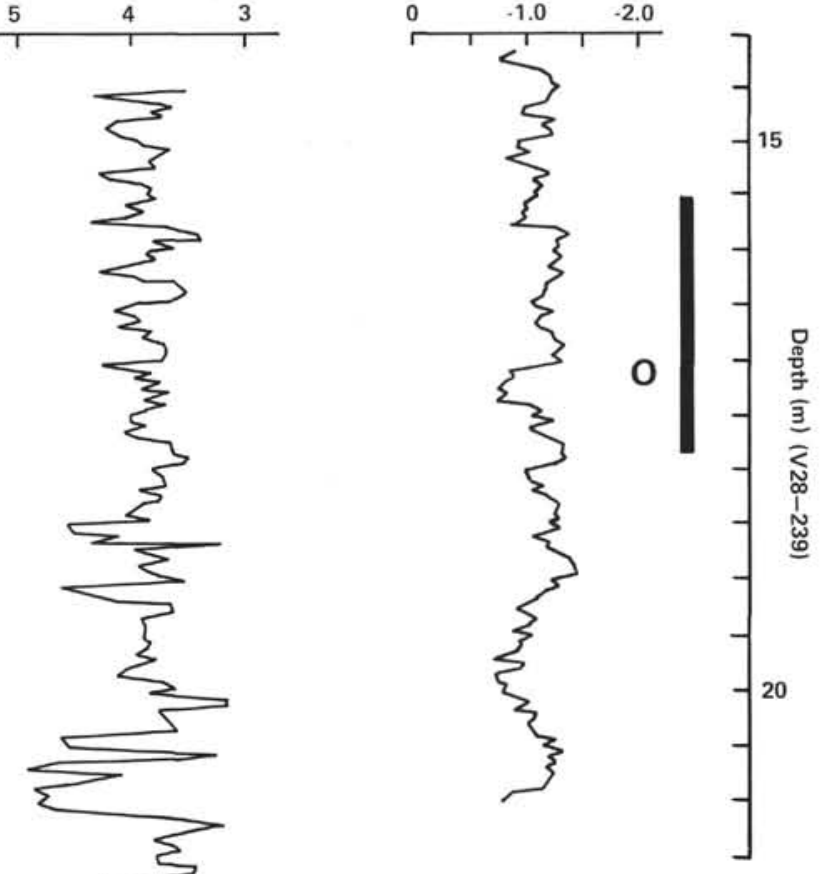

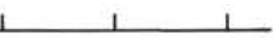

1. The climate-ocean system, expressed as lithologic parameters, clearly shows a cyclic mode of variation. The gross lithology is a sequence of alternating white pelagic-calcareous ooze and dark muds rich in ice-rafted debris. Composition of the acid-insoluble coarse fraction and geographic position of Hole 552A suggest Greenland as the most probable origin of the ice-rafted debris.

2 . The cyclic nature of the sediments extends to the included organic material. The glacial muds, with a relatively high content of organic carbon of a reworked character, reflect the lowered sea levels and increased con- 


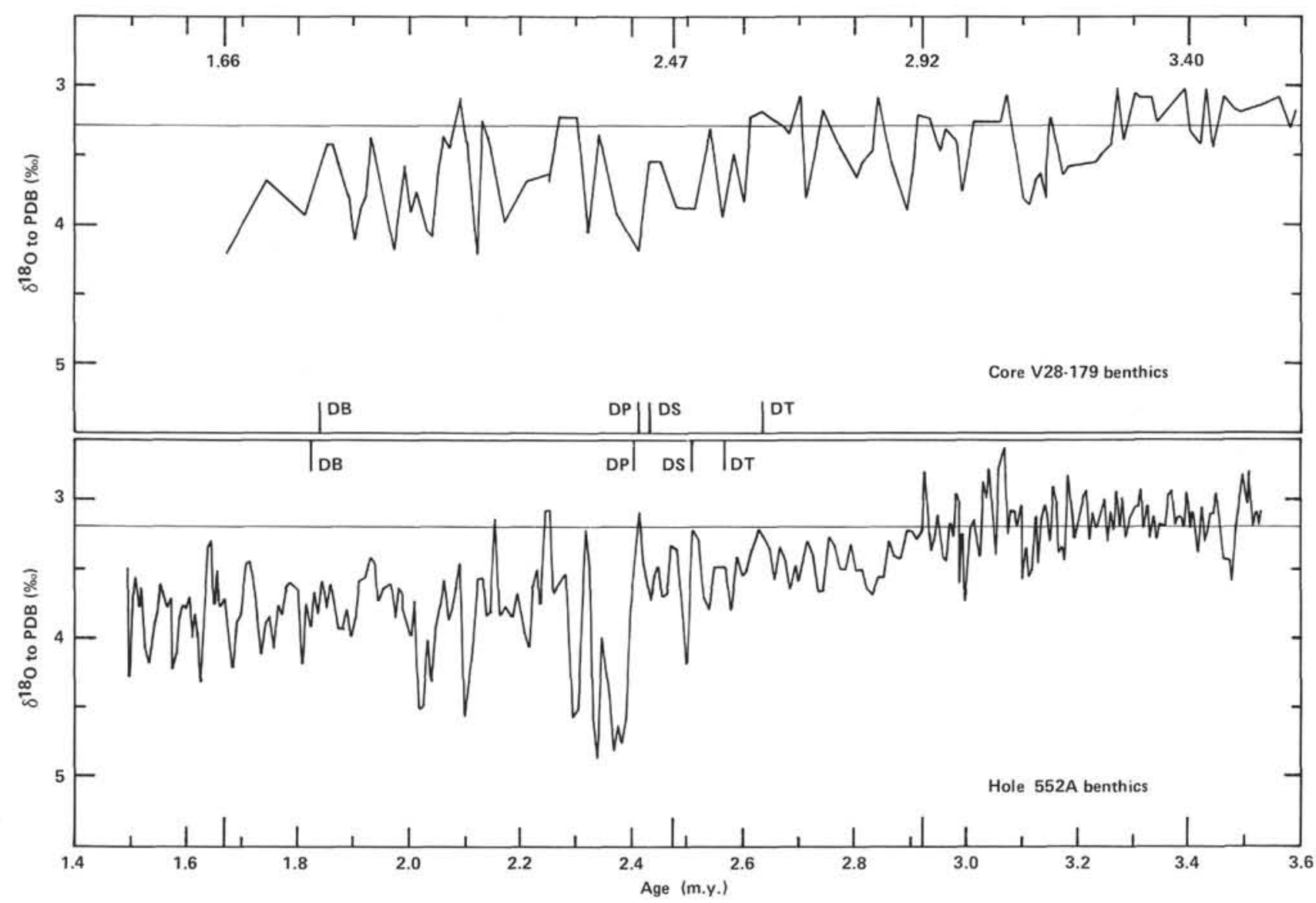

Figure 8. Oxygen isotope data of Hole 552A (Cores 7-12) plotted on an age scale of linear adjustment between magnetic reversals. The oxygen isotope record for Pacific core V28-179 (Shackleton and Opdyke, 1977) is plotted at the same age scale for comparison; time control is shown - 1.66 m.y., top Olduvai N subchron; 2.47 m.y. ago, base Matuyama R chron; 2.92 m.y., top Kaena R subchron; 3.40 m.y., base Gauss N chron. Nannofossil extinction horizons determined for both cores: DT, Discoaster tamalis; DS, D. surculus; DP, D. pentaradiatus; $\mathrm{DB}, D$. brouweri. Horizontal lines indicate the present position of $\mathrm{O}^{18}$ equilibrium.

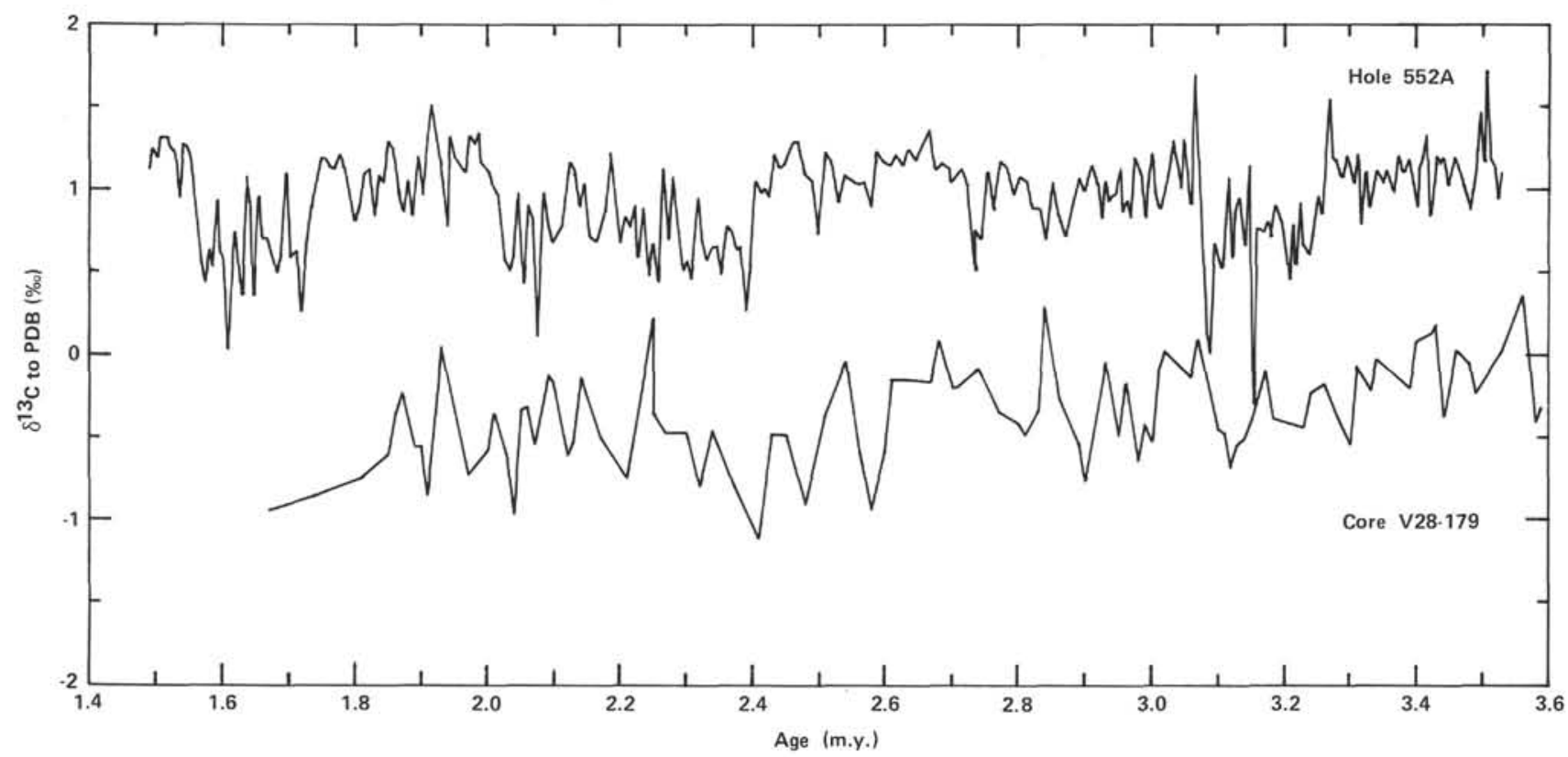

Figure 9. Carbon isotope records for Hole 552A (Cores 7-12) and Pacific core V28-179. Both records are plotted on the same ${ }^{13} \mathrm{C}$ and age scales. 
tinental erosion of glacial episodes. The enhanced levels of organic phosphorus in organic material of the interglacial stages suggest a pelagic-marine source.

3. The carbonate and coarse fraction data reflect the variation of the calcareous components produced under the rapidly changing climatic regimes of the past $2.4 \mathrm{~m}$.y. Spikes of low coarse fraction carbonate may indicate particularly intense cold periods with extensive ice cover and resulting low productivity in the northeastern Atlantic. In the opposite sense, episodes of particular warmth are suggested by isolated occurrences of temperate diatoms.

4. In the upper part of the section, DSDP Hole 552A preserves the whole of the standard oxygen isotope record of the past million years. We have extended the isotope stage stratigraphy to interglacial stage 25 which includes the base of the Jaramillo subchron at $0.98 \mathrm{~m}$.y. ago.

5. Oxygen isotope analysis of the sequence together with nannofossil and magnetostratigraphic time zontrol indicate that the first major glacial event, as represented by coincident ice volume and ice-rafting horizons, occurs at 2.37 m.y. ago, well before the Pliocene/Pleistocene boundary. These data do not support the notion of significant northern hemisphere glaciation prior to 2.5 m.y. ago.

6. The carbon isotope record shows that Hole 552A has been bathed by a water mass with characteristics similar to those of present-day North Atlantic Deep Water at least since $3.5 \mathrm{~m} . \mathrm{y}$. ago and that quasi-cyclic alternations in the intensity of its production were occurring during the Pliocene prior to the onset of extensive glaciation in the northern hemisphere.

\section{ACKNOWLEDGMENTS}

The authors would like to thank the staff at DSDP for the opportunity to participate on Leg 81 and for their encouragement to complete this study. The good fellowship and stimulating collaboration of the shipboard scientific party will serve as a model for future cruises. Our sincere thanks to the captain and crew of The D/V Glomar Challenger for a job well done under the trying circumstances of the North Atlantic.

\section{REFERENCES}

Backman, J., 1979. Pliocene biostratigraphy of DSDP Sites 111 and 116 from the North Atlantic Ocean and the age of northern hemisphere glaciation. Stockholm Contrib. Geol., 32:113-137.

Backman, J., and Shackleton, N. J., 1983. Quantitative biochronology of Pliocene and early Pleistocene calcareous nannofossils from the Atlantic, Indian and Pacific Oceans. Mar. Micropaleontol., 8: 141-170.

Backman, J., Shackleton, J. J. and Tauxe, L., 1983. Quantitative nannofossil correlation to open ocean deep sea sections from the PlioPleistocene boundary at Vrica, Italy. Nature, 304:156-158.

Berggren, W. A., 1972. Late Pliocene-Pleistocene glaciation. In Laughton, A. S., Berggren, W. A., et al., Init. Repts. DSDP, 12: Washington (U.S. Govt. Printing Office), 953-963.

Berggren, W. A., Kent, D. V., and Van Couvering, J., in press. Neogene geochronology and chronostratigraphy. J. Geol. Soc. London.
Donn, W. L., and Ninkovitch, D., 1980. Rate of Cenozoic explosive volcanism in the North Atlantic Ocean inferred from deep sea cores. J. Geophys. Res., 85:5455-5460.

Goree, W. S., and Fuller, M., 1976. Magnetometers using RF-driven squids and their application in rock magnetism and paleomagnetism. Rev. Geophys. Space Phys., 14:591-608.

Harrison, R. K., Knox, R. W. O'B., and Morton, A. C., 1979. Petrography and mineralogy of volcanogenic sediments from DSDP Leg 48, southwest Rockall Plateau, Site 403 and 404. In Montadert, L., Roberts, D. G., et al., Init. Repts. DSDP., 48: Washington (U.S. Govt. Printing Office), 771-785.

Heiken, G., 1975. An atlas of volcanic ash. Smithsonian Contrib. Earth Sci., 12:1-101.

Hodell, D. A., Kennett, J. P., and Leonard, K. A., 1983. Climatically induced changes in vertical water mass structure of the Vema Channel: Evidence from Deep Sea Drilling Project Holes 516A, 517, and 518. In Barker, P. F., Johnson, D. A., et al., Init. Repts. $D S D P, 72$ : Washington (U.S. Govt. Printing Office), 907-920.

Hodell, D. A., Williams, D. F., and Kennett, J. P., in press. Reorganization of deep vertical water mass structure in Vema Channel at 3.2 Ma: faunal and isotopic evidence from DSDP Leg 72. Geol. Soc. Am. Bull.

Kroopnick, P., 1980. The distribution of ${ }^{13} \mathrm{C}$ in the Atlantic Ocean. Earth Planet. Sci Lett., 49:469-484.

McCave, I. N., Lonsdale, P. F., Hollister, C. D., and Gardner, W. D., 1980. Sediment transport over the Hatton and Gardar Contourite Drifts. J. Sediment, Petrol., 50:1049-1062.

Müller, G., and Gastner, M., 1971. The "Karbonate-Bombe," a simple device for the determination of the carbonate content in sediments, soils, and other materials. N. Jb. Miner, Mh., 10:466-469.

Poore, R. Z., 1981. Temporal and spatial distribution of ice-rafted mineral grains in Pliocene sediments of the North Atlantic: Implications for late Cenozoic climatic history. SEPM Spec. Publ., 32: 505-515.

Prell, W. L., 1982. A reevaluation of the initiation of northern hemisphere glaciation at $3.2 \mathrm{~m} . \mathrm{y}$.: New isotope evidence. Geol. Soc. Am., (95th Ann. Mtg.), p. 592 (Abstract with program).

Ruddiman, W. F., and Glover, L. K., 1972. Vertical mixing of ice-rafted volcanic ash in North Atlantic sediments. Geol. Soc. Am. Bull., 83:2817-2836.

Ruddiman, W. F., and McIntyre, A., 1976. Northeast Atlantic paleoclimatic changes over the past 600,000 years. Mem. Geol. Soc. Am., 145:111-146.

Schrader, H. J., and Fenner, J., 1976. Norwegian diatom biostratigraphy and taxonomy. In Talwani, M., Udintsev., G., et al., Init Repts. DSDP, 38: Washington (U.S. Govt. Printing Office), 921-1098.

Shackleton, N. J., Backman, J., Zimmermann, H. B., Kent, D. V. Hall, M. A., Roberts, D. G., Schnitker, D., Baldauf, J. G., Desprairies, A., Homrighausen, R., Huddlestun, P., Keene, J. B., Kaltenback, A. J., Krumsiek, K. A. O., Morton, A. C., Murray, J. W., and Westberg-Smith, J., 1984. Oxygen isotope calibration of the onset of icerafting in DSDP Site 552A: History of glaciation in the North Atlantic region. Nature, 307:620-623.

Shackleton, N. J., and Opdyke, N. D., 1973. Oxygen isotope and paleomagnetic stratigraphy of equatorial Pacific core V28-238: Oxygen isotope temperatures and ice volumes on a $10^{5}$ year and $10^{6}$ year scale. Quat. Res., 3:39-55. 1976. Oxygen-isotope and paleomagnetic stratigraphy of $\mathrm{Pa}$ cific core V28-239, late Pliocene to latest Pleistocene. Mem. Geol. Soc. Am., 145:449-464. , 1977. Oxygen isotope and paleomagnetic evidence for early Northern Hemisphere glaciation. Nature, 270: 216-219.

Sylvester, A. G., 1976. Petrography of volcanic ashes in deep-sea cores near Jan-Mayen Island: Sites 338, 345-350, DSDP Leg 38. In Talwani, M., Udintsev, G., Init. Repts. DSDP, Suppl. to Vols. 38, 39, 40, and 41: Washington (U.S. Govt. Printing Office), 101-106.

Date of Acceptance: January 30, 1984 